\title{
Gaussian mixture probability hypothesis density filter for multipath multitarget tracking in over-the-horizon radar
}

\author{
Yong Qin ${ }^{1 *}$, Hong Ma ${ }^{1}$, Jinfeng Chen ${ }^{1}$ and Li Cheng ${ }^{1,2}$
}

\begin{abstract}
Conventional multitarget tracking systems presume that each target can produce at most one measurement per scan. Due to the multiple ionospheric propagation paths in over-the-horizon radar (OTHR), this assumption is not valid. To solve this problem, this paper proposes a novel tracking algorithm based on the theory of finite set statistics (FISST) called the multipath probability hypothesis density (MP-PHD) filter in cluttered environments. First, the FISST is used to derive the update equation, and then Gaussian mixture (GM) is introduced to derive the closed-form solution of the MP-PHD filter. Moreover, the extended Kalman filter (EKF) is presented to deal with the nonlinear problem of the measurement model in OTHR. Eventually, the simulation results are provided to demonstrate the effectiveness of the proposed filter.
\end{abstract}

Keywords: Over-the-horizon radar (OTHR), Multipath multitarget tracking, Probability hypothesis density filter, Finite set statistics (FISST)

\section{Introduction}

Over-the-horizon radar (OTHR) exploits skywave propagation of high-frequency signals to detect and track targets, which are different from the conventional radar. It has received wide attention because of its wide area surveillance, long detection range, strong anti-stealth ability, the capability of the long early warning time, and so on. In OTHR, a significant problem is the effect of multipath propagation, which causes multiple detections via different propagation paths for a target with missed detections and false alarms at the receiver [1-6]. Nevertheless, the conventional tracking algorithms, such as probabilistic data association (PDA) [7-9], presume that a single-measurement per target, it may consider the other measurements of the same target as clutter, and multiple tracks are produced when a single target is present. Therefore, these methods cannot effectively solve the multipath propagation problem.

In order to solve the multipath propagation problem of OTHR, lots of algorithms have been proposed. Such

\footnotetext{
* Correspondence: qinyong1208@163.com

${ }^{1}$ School of Electronic Information and Communications, Huazhong University of Science and Technology, Wuhan 430074, China

Full list of author information is available at the end of the article
}

as the multipath viterbi data association (MVDA) [10] and modified probabilistic data association (MPDA) [11]. However, these conventional algorithms involve data association problem and the complexity of the computation. In addition, it is noted that most of the existing algorithms in OTHR focus on a single target. Therefore, the multitarget tracking algorithm in OTHR is still an open problem.

Most of the conventional algorithms about multitarget tracking, such as multiple hypothesis tracker (MHT) [12], joint probabilistic data association (JPDA) [13, 14], and probability hypothesis density (PHD) filter [15], assume the following measurement model: (1) every target produces at most one measurement and (2) any measurement is produced by a target or clutter. In this paper, we consider the measurement model which satisfies these assumptions as standard measurement model. However, many measurement models in real-life target tracking scenarios do not satisfy these prerequisite of assumption, which are treated as nonstandard measurement model. Recently, the multiple detection joint probabilistic data association (MD-JPDA) filter [16] based on the JPDA framework was proposed to deal with the multiple detection targets, which can apply to OTHR.

\section{Springer}

(c) 2015 Qin et al. Open Access This article is distributed under the terms of the Creative Commons Attribution 4.0 International License (http://creativecommons.org/licenses/by/4.0/), which permits unrestricted use, distribution, and reproduction in any medium, provided you give appropriate credit to the original author(s) and the source, provide a link to the Creative Commons license, and indicate if changes were made. 
However, this algorithm involves complexity data association. To solve this problem of multitarget tracking, more effective methods should be taken into account to implement in OTHR.

In 2009, Mahler derived a series of "second-generation" $\mathrm{PHD} /$ cardinalized probability hypothesis density (CPHD) filters [17] which addressed the tracking problems for nonstandard multitarget measurement model, such as extended targets [18-21], unresolved target [22], unknown clutter [23], and superpositional sensors [24]. As for the multipath propagation in OTHR, the receiver can obtain more than one measurement from the same target, which means that it violates the second assumption of the standard measurement model. Therefore, the multipath tracking model in OTHR is one of the nonstandard measurement models, such that conventional multitarget tracking algorithms would no longer be applicable.

To solve the multipath propagation problem of multitarget, we propose a novel tracking algorithm based on the theory of finite set statistics (FISST) called the multipath probability hypothesis density (MP-PHD) filter in OTHR. First, inspired by Mahler's work in [17], the FISST is used to derive the update equation of the MPPHD filter. Then, a method of Gaussian mixture (GM) is introduced to derive the closed-form solution of the MP-PHD filter. In addition, the extended Kalman filter (EKF) is presented to deal with the nonlinear problem of the measurement model in OTHR. The simulation results demonstrate that the MP-PHD filter can accurately estimate the target state and the target number for low detection probability under the multitarget tracking circumstance.

This paper is organized as follows. Section 2 presents the problem formulation for OTHR. Section 3 then formulates the random finite set (RFS) measurement model for the OTHR tracking problem and derives the MPPHD filter in OTHR based on the FISST. A closed-form implementation of MP-PHD filter is presented in Section 4. In Section 5, the simulation results are presented. Finally, some conclusions and future works are discussed in Section 6.

\section{Problem formulation}

\subsection{Dynamic model}

The geometry of the target and OTHR system is depicted in Fig. 1. The target state vector of OTHR at time $k$ is defined by $x_{k}=[\rho(k), . \rho(k), b(k), . b(k)]^{\prime}, x_{k} \in \tilde{J}$, where $\rho(k)=\rho_{1}(k), . \rho(k), b(k)$, and.$b(k)$ are the ground range, range rate, bearing, and bearing rate and $\tilde{s}$ is the space of the ground coordinates, the dash denotes transposition.

Since the distance between the receiver and the targets is large, we usually assume that the state equation of OTHR is linear and discrete-time. It could be modeled as

$$
x_{k}=F x_{k-1}+u_{k-1}
$$

where $u_{k-1}$ is a zero mean, white Gaussian noise with covariance $Q_{k-1}$, and the state transition matrix $F$ is given by

$$
F=\left[\begin{array}{llll}
1 & T & 0 & 0 \\
0 & 1 & 0 & 0 \\
0 & 0 & 1 & T \\
0 & 0 & 0 & 1
\end{array}\right]
$$

where $T$ is the sampling period.

\subsection{Measurement model of OTHR}

OTHR achieves beyond the line-of-sight horizon target tracking by exploiting skywave propagation of highfrequency signals via the ionospheric, and depending on

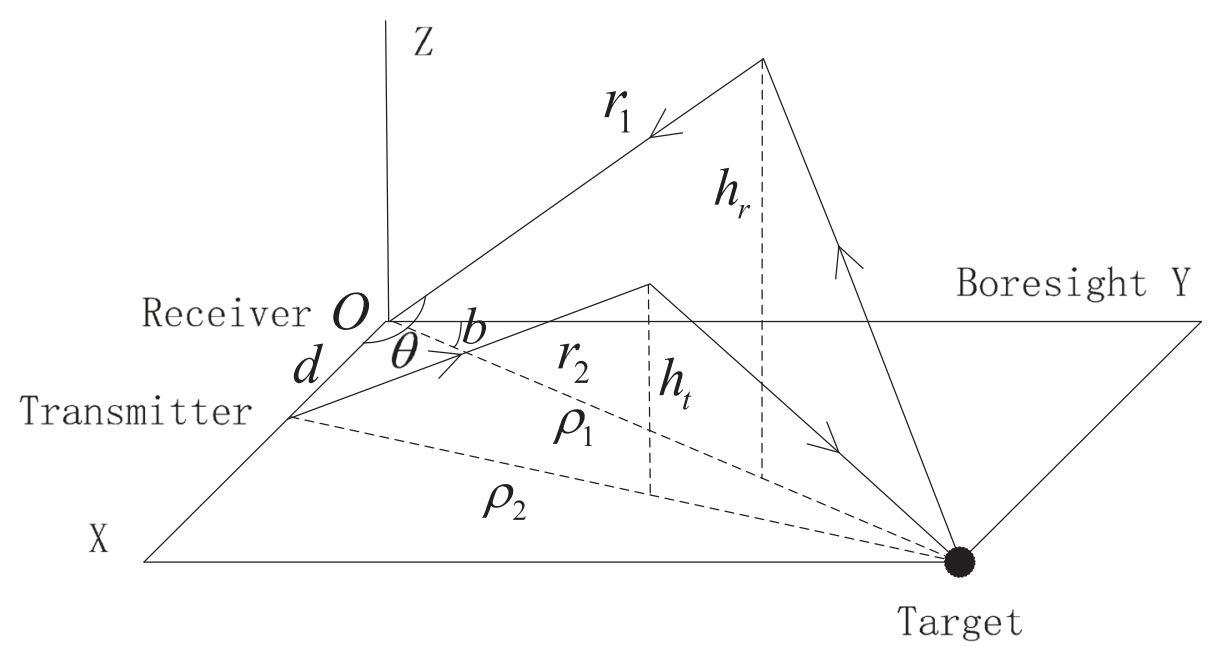

Fig. 1 Geometry of a planar OTHR measurement model with a collocated transmitter and receiver. $h_{r}$ and $h_{t}$ denote reflecting ionosphere heights 
the particular ionospheric propagation conditions, there may be several propagation paths. As shown in Fig. 1, the OTHR signal reflects from the transmitter to the target by an ionospheric at height $h_{\mathrm{t}}$, then the signal reflects from the target to the receiver by an ionospheric at height $h_{\mathrm{r}}$. As is well known, there are many ionospheric layers present in the atmosphere, and we suppose that there are two ionospheric layers $\mathrm{E}$ and $\mathrm{F}$ for simplicity, with constant heights $h_{\mathrm{E}}$ and $h_{\mathrm{F}}$ (this suppose is the same as other algorithms in OTHR). Therefore, there are four propagation modes: mode EE (transmit on $\mathrm{E}$ and receive on $\mathrm{E}$ ); mode $\mathrm{EF}$ (transmit on $\mathrm{E}$ and receive on $\mathrm{F}$ ); mode $\mathrm{FE}$ (transmit on $\mathrm{F}$ and receive on $\mathrm{E}$ ); and mode FF (transmit on $\mathrm{F}$ and receive on F). It means that the receiver can obtain more than one measurement from the same target at one time.

The OTHR measurements consist of a slant range $\operatorname{Rg}=r_{1}+r_{2}$, Doppler $f_{d}$, and $\mathrm{Az}=\pi / 2-\theta(\theta$ is the azimuth as shown in Fig. 1) of the form $z_{k}=\left[\operatorname{Rg}(k), f_{d}(k), \mathrm{Az}(k)\right]^{\prime}, z_{k} \in \mathcal{G}$, where $\mathcal{G}$ is the space of the slant coordinates.

The measurement model of OTHR involves the mapping from the ground coordinates $(\rho, . \rho, b, . b)$ to the slant coordinates $\left(\mathrm{Rg}, f_{d}, \mathrm{Az}\right)$. From Fig. 1 , it can be shown that the mapping is expressed as [2]

$$
\left\{\begin{array}{c}
\mathrm{Rg}=r_{1}+r_{2} \\
f_{d}=\frac{\dot{\rho}}{4}\left\{\frac{\rho}{r_{1}}+\frac{\eta}{r_{2}}\right\} \\
\mathrm{Az}=\sin ^{-1}\left\{\rho \sin (b) /\left(2 r_{1}\right)\right\}
\end{array}\right.
$$

where

$$
\begin{aligned}
& r_{1}=\sqrt{(\rho / 2)^{2}+h_{r}^{2}} \\
& r_{2}=\sqrt{(\rho / 2)^{2}-d \rho \sin (b) / 2+(d / 2)^{2}+h_{t}^{2}} \\
& \eta=\rho-d \sin (b)
\end{aligned}
$$

Then, the measurement model of OTHR can be expressed as [11]

$$
z_{k}= \begin{cases}h_{1}\left(x_{k}\right)+w_{k, 1} & \text { if mode EE } \\ h_{2}\left(x_{k}\right)+w_{k, 2} & \text { if mode EF } \\ h_{3}\left(x_{k}\right)+w_{k, 3} & \text { if mode FE } \\ h_{4}\left(x_{k}\right)+w_{k, 4} & \text { if mode FF } \\ \text { clutter } & \text { otherwise }\end{cases}
$$

where $x_{k}$ is the state variable; $w_{k, i}$ is the zero mean, white Gaussian noise with known covariance $R_{k, i}$, and $h_{i}(\cdot)$ is the nonlinear measurement function of $i$ th propagation mode, $i=1,2,3,4$.

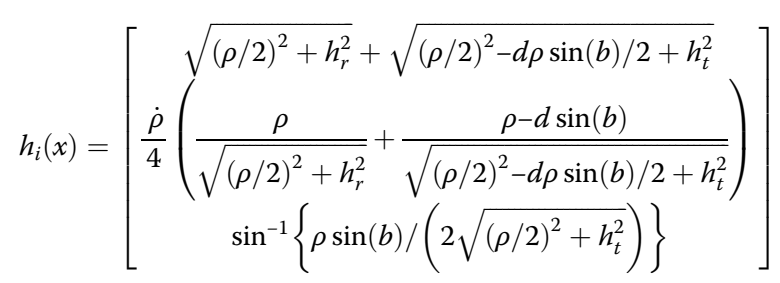

where, for a given propagation mode $i, h_{t}$ and $h_{r}$ must be replaced by the virtual ionosphere heights, where the details can be found in [11].

\section{The RFS model and PHD filter implementation in OTHR}

In the standard PHD filter, it is presumed that a target produces at most one measurement. Due to the multipath effect of OTHR, a single target may generate several measurements. Therefore, the measurement model of the MP-PHD filter proposed in this paper is different from the standard PHD filter. In this section, we first introduce the RFS measurement model for OTHR and then derive the update equation of the MP-PHD filter based on the theory of FISST.

\subsection{The RFS measurement model for OTHR}

At each time $k$, a finite set of measurements of OTHR, denoted by $Z^{k}=\left\{z_{k, 1}, z_{k, 2}, \cdots z_{k, N_{k}}\right\}$, where $z_{k, 1}, z_{k, 2}, \cdots$ $z_{k, N_{k}}$ are the received measurements at time $k$ and $N_{k}$ is the number of measurements. Since the measurements include several detections from targets and clutter, the collection of measurements of OTHR can be modeled by RFS

$$
Z^{k}=\Theta_{k, 1}\left(x_{k}\right) \cup \Theta_{k, 2}\left(x_{k}\right) \cup \Theta_{k, 3}\left(x_{k}\right) \cup \Theta_{k, 4}\left(x_{k}\right) \cup \Gamma_{k}
$$

where $\Theta_{k, i}\left(x_{k}\right), i=1, \cdots, 4$ denotes the measurement originated from the $i$ th propagation path and $\Gamma_{k}$ denotes the RFS of clutter. It is presumed that conditional on $x_{k}$, $\Theta_{k, i}\left(x_{k}\right), i=1, \cdots, 4$ and $\Gamma_{k}$ are independent RFSs.

\subsection{The update equation of MP-PHD filter in OTHR}

Note that both the MP-PHD filter and the standard PHD filter recursion require two steps: prediction and update. In the following subsection, we only derive the update equation according to the above RFS measurement model since the prediction step of the MP-PHD filter is identical to the standard PHD filter.

In ref. [17], the probability generating functional (PGFL) of update equation for the multitarget Bayes filter can be written as 


$$
G_{k \mid k}[h]=\frac{\frac{\delta F}{\delta Z_{k}}[0, h]}{\frac{\delta F}{\delta Z_{k}}[0,1]}
$$

where $F[g, h]$ is two-variable PGFL as

$$
\begin{aligned}
& F[g, h]=\int h^{X} \cdot G_{k}[g \mid X] \cdot f_{k \mid k-1}\left(X \mid Z^{(k-1)}\right) \delta X \\
& G_{k}[g \mid X]=\int g^{Z} \cdot f_{k}(Z \mid X) \delta Z
\end{aligned}
$$

where $f_{k \mid k-1}\left(X \mid Z^{(k-1)}\right)$ is the predicted multitarget distribution, $f_{k}(Z \mid X)$ is the multitarget likelihood function and $h^{X}$ is defined by $h^{X}=1$ when $X=\varnothing$ and $h^{X}=\Pi_{x \in X} h(x)$ otherwise. The update PHD filter can be given by

$$
D_{k \mid k}(x)=\frac{\delta G_{k \mid k}}{\delta x}[1]
$$

Therefore, the update equation for MP-PHD filter can be derived by the following procedures:

1) Derive the PGFL $G_{k}[g \mid X]$ by using the OTHR measurements and exploiting Eq. (11) to derive a closed form of $F[g, h]$

2) Derive the derivatives of $F[g, h]$ by using Eq. (10) to derive a closed form of $G_{k \mid k}[h]$

3) According to Eq. (11), derive a closed-form update for the MP-PHD filter in OTHR

In the following part of this subsection, we will present the details of the derivation. First, we derive the PGFL $G_{k}[g \mid X]$ according to the OTHR measurements in Eq. (9). Since $\Theta_{k}(x)=\Theta_{k, 1}(x) \cup \Theta_{k, 2}(x) \cup \Theta_{k, 3}(x) \cup \Theta_{k, 4}(x)$, the PGFL $G_{k}[g \mid x]$ of $\Theta_{k}(x)$ is

$$
\begin{aligned}
G_{k}[g \mid x]= & \prod_{i=1}^{4} G_{k, i}[g \mid x]=\prod_{i=1}^{4}\left(1-p_{D, k}(x)+p_{D, k}(x) p_{g, i}(x)\right) \\
= & \left(1-p_{D, k}(x)\right)^{4}+\left(1-p_{D, k}(x)\right)^{3} p_{D, k}(x)\left(\sum_{i=1}^{4} p_{g, i}(x)\right) \\
& +\left(1-p_{D, k}(x)\right)^{2} p_{D, k}^{2}(x)\left(\sum_{i=1}^{3} \sum_{j=i+1}^{4} p_{g, i}(x) p_{g, j}(x)\right) \\
& +\left(1-p_{D, k}(x)\right) p_{D, k}^{3}(x)\left(\sum_{i=1}^{2} \sum_{j=i+1}^{3} \sum_{m=j+1}^{4} p_{g, i}(x) p_{g, j}(x) p_{g, m}(x)\right) \\
& +p_{D, k}^{4}(x)\left(\sum_{i=1} \sum_{j=2} \sum_{m=3} \sum_{n=4} p_{g, i}(x) p_{g, j}(x) p_{g, m}(x) p_{g, n}(x)\right)
\end{aligned}
$$

where $p_{g, i}(x)=\int g(z) g_{k, i}(z \mid x) d z, p_{D, k}(x)$ is the detection probability, and $g_{k, i}(z \mid x)$ is the likelihood of the $i$ th propagation path. Consequently, if we abbreviate $q_{D, k}(x)=1-p_{D, k}(x)$, Eq. (11) can be summarized as follows:

$$
\begin{aligned}
F[g, h]= & e^{\lambda[g]]-\lambda} \cdot G_{k \mid k-1}\left[h \left(q_{D, k}^{4}+q_{D, k}^{3} p_{D, k}\left(\sum_{i=1}^{4} p_{g, i}\right)\right.\right. \\
& +q_{D, k}^{2} p_{D, k}^{2}\left(\sum_{i=1}^{3} \sum_{j=i+1}^{4} p_{g, i} p_{g, j}\right) \\
& +q_{D, k} p_{D, k}^{3}\left(\sum_{i=1}^{2} \sum_{j=i+1}^{3} \sum_{m=j+1}^{4} p_{g, i} p_{g, j} p_{g, m}\right) \\
& \left.\left.+p_{D, k}^{4}\left(\sum_{i=1} \sum_{j=2} \sum_{m=3} \sum_{n=4} p_{g, i} p_{g, j} p_{g, m} p_{g, n}\right)\right)\right]
\end{aligned}
$$

where $c[g]=\int g(z) c_{k}(z) d z \quad$ and $\quad G_{k \mid k-1}[h]=\int h^{X} f_{k \mid k-1}$ $\left(X \mid Z^{(k-1)}\right) \delta X$.

To derive a closed-form update equation for the multipath multitarget PHD filter, we assume that the predicted multitarget distribution is a Poisson process:

$$
G_{k \mid k-1}[h]=e^{\mu s[h]-\mu}
$$

where $s[h]=\mu^{-1} \int h(x) D_{k \mid k-1}(x) d x$ and $\mu=\int D_{k \mid k-1}(x) d x$. Thus, Eq. (15) can be written as

$$
\begin{aligned}
F[g, h]= & \exp \left(\left(\lambda c[g]-\lambda+\mu s\left[h q_{D, k}^{4}\right]+\mu s\left[h q_{D, k}^{3} p_{D, k}\left(\sum_{i=1}^{4} p_{g, i}\right)\right]\right.\right. \\
& +\mu s\left[h q_{D, k}^{2} p_{D, k}^{2}\left(\sum_{i=1}^{3} \sum_{j=i+1}^{4} p_{g, i} p_{g, j}\right)\right] \\
& +\mu s\left[h q_{D, k} p_{D, k}^{3}\left(\sum_{i=1}^{2} \sum_{j=i+1}^{3} \sum_{m=j+1}^{4} p_{g, i} p_{g, j} p_{g, m}\right)\right] \\
& \left.+\mu s\left[h p_{D, k}^{4}\left(\sum_{i=1} \sum_{j=2} \sum_{m=3} \sum_{n=4} p_{g, i} p_{g, j} p_{g, m} p_{g, n}\right)\right]-\mu\right)
\end{aligned}
$$

Next, we deduce the formula for $\frac{\delta F}{\delta Z}[0, h]$. Note that we set $g=0$ because the formula for $\frac{\delta F}{\delta Z}[g, h]$ is very cumbersome without setting it.

Lemma 1. The derivatives of $F[g, h]$ is given by the formula

$$
\frac{\delta F}{\delta Z}[0, h]=F[0, h] \cdot \prod_{Z} \cdot \sum_{\wp \subset Z} \prod_{W \in \wp} d_{W}[0, h]
$$

where the notation ' $\circ \angle Z$ ' is shorthand for " 8 partitions $Z$ into cells $W^{\prime \prime}, \Pi_{Z}=\Pi_{z \in Z} \lambda c(z)$, 


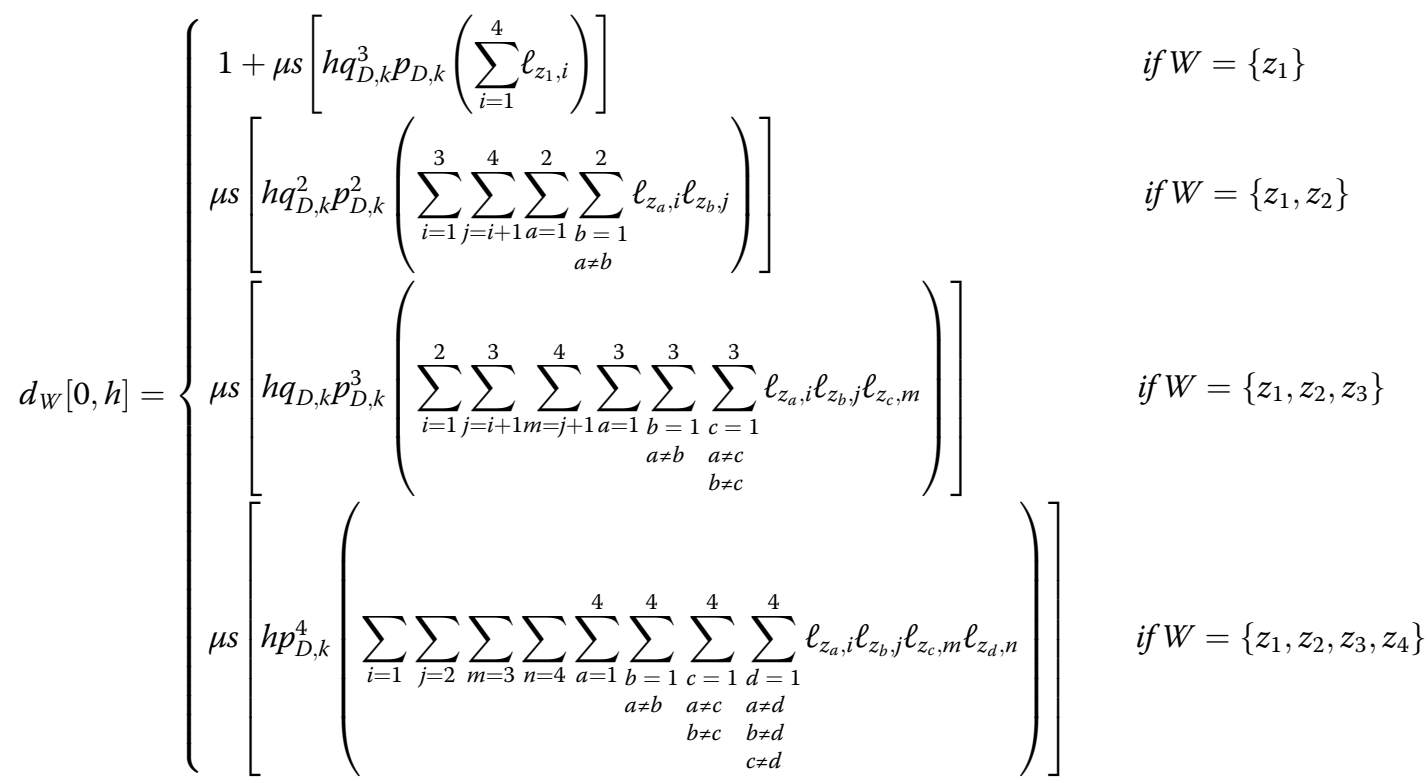

and where

$$
\ell_{z, i}(x)=\frac{g_{k, i}(z \mid x)}{\lambda c(z)} \quad i=1, \cdots, 4 .
$$

See Appendix 1 for the proof of Lemma 1.

Lemma 2. The multipath posterior PGFL $G_{k \mid k}[h]$ is given as

$$
G_{k \mid k}[h]=F_{0}[h] \cdot \frac{\sum_{\wp\left\llcorner Z_{k}\right.} \prod_{W \in \wp^{\prime}} d_{W}[0, h]}{\sum_{\wp^{\prime} \angle Z_{k}} \prod_{W \in \wp^{\prime}} d_{W}[0,1]}
$$

where

$$
F_{0}[h]=\exp \left(\mu s\left[(h-1) q_{D, k}^{4}\right]\right)
$$

and $d_{W}[0, h]$ is the Eq. (19).

See Appendix 2 for the proof of Lemma 2.

According to Lemmas 1 and 2, we can obtain the MP-PHD filter update equation in Proposition 1 as follows:

Proposition 1. The update equation for the MP-PHD filter is

$$
D_{k \mid k}\left(x \mid Z^{(k)}\right) \cong L_{Z_{k}}\left(x \mid Z^{(k-1)}\right) \cdot D_{k \mid k-1}\left(x \mid Z^{(k-1)}\right)
$$

where

$$
L_{Z_{k}}\left(x \mid Z^{(k-1)}\right)=q_{D, k}^{4}(x)+\sum_{\wp\left\llcorner Z_{k}\right.} \omega_{\wp} \cdot \sum_{W \in \wp} \frac{b_{W}}{d_{W}}
$$

here

$$
\omega_{\mathfrak{S}}=\frac{\prod_{W \in \mathcal{S}} d_{W}}{\sum_{\mathcal{S}^{\prime} \angle Z_{k}} \prod_{W \in \mathcal{G}^{\prime}} d_{W}}
$$


where

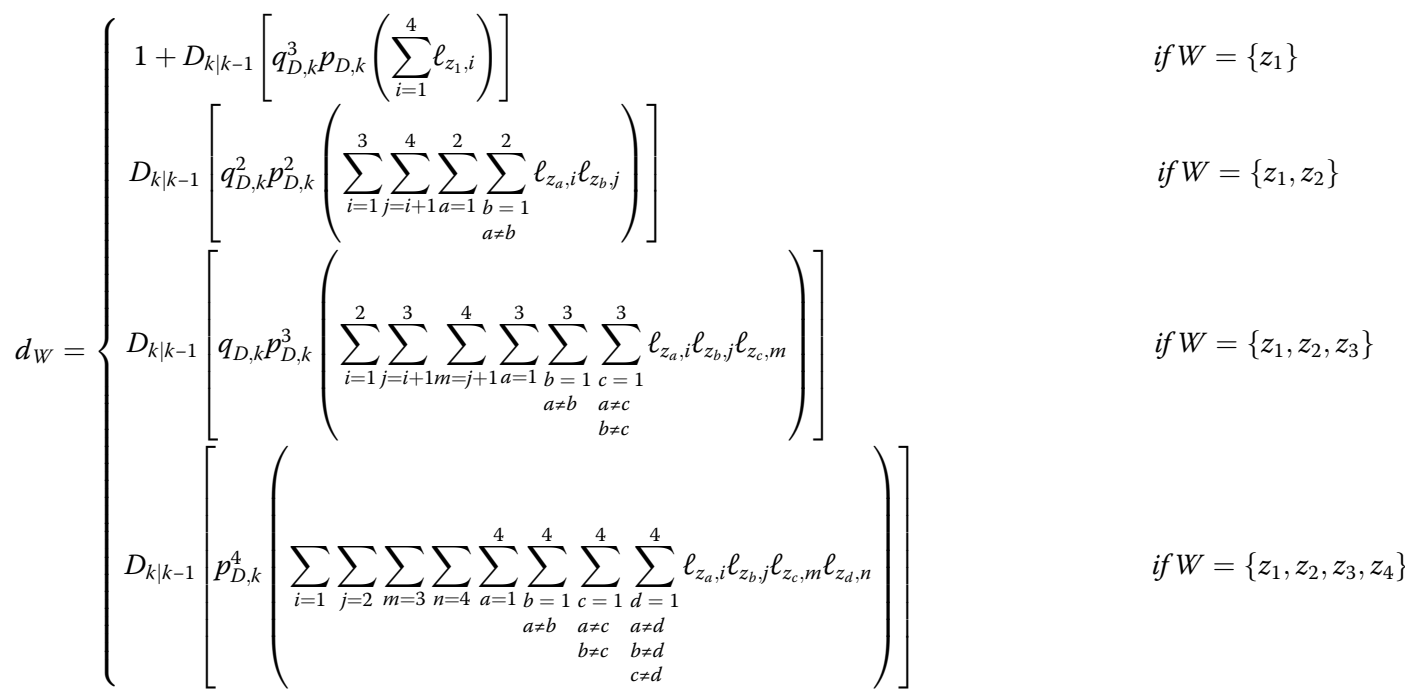

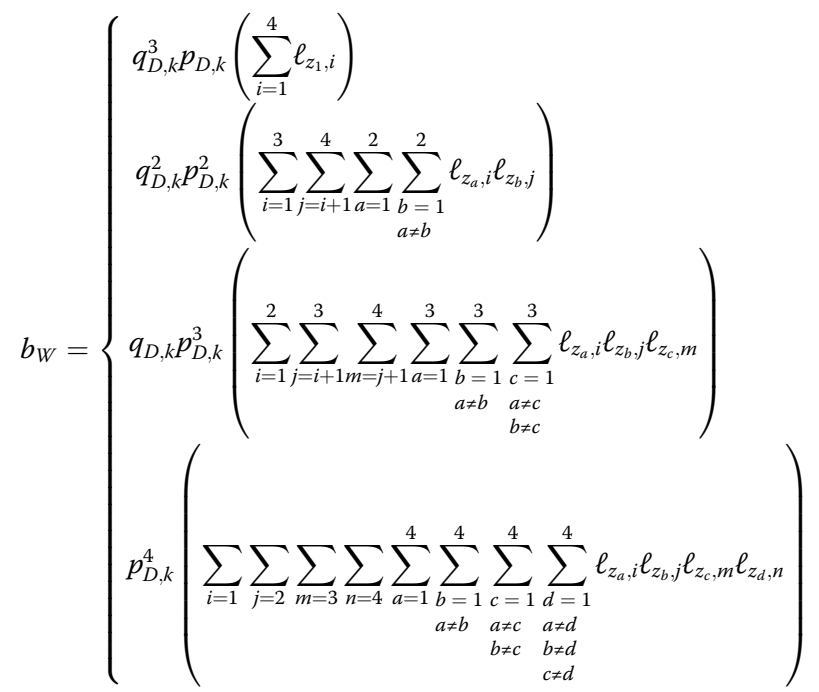

$$
\begin{aligned}
& \text { if } W=\left\{z_{1}\right\} \\
& \text { if } W=\left\{z_{1}, z_{2}\right\} \\
& \text { if } W=\left\{z_{1}, z_{2}, z_{3}\right\} \\
& \text { if } W=\left\{z_{1}, z_{2}, z_{3}, z_{4}\right\}
\end{aligned}
$$

See Appendix 3 for the proof of Proposition 1.

\section{Gaussian mixture implementation of the MP-PHD filter}

According to the GM implemented in the standard PHD filter in [25], a GM-PHD filter recursion can be derived for the multipath target tracking. Due to the MP-PHD filter prediction equation for the OTHR are identical to the standard PHD filter, the GM prediction equation of MP-PHD filter are the same as the standard GM-PHD filter, where the details can be found in [25]. Therefore, in this section, we only consider the GM update equation of the MP-PHD filter.

\subsection{Gaussian mixture MP-PHD}

To derive a closed-form solution to the update equation of the MP-PHD filter, we assume that each target follows a linear-Gaussian transition and measurement model, i.e.,

$$
\begin{aligned}
& f_{k \mid k-1}(x \mid \zeta)=N\left(x ; F_{k \mid k-1} x_{k \mid k-1}, Q_{k \mid k-1}\right) \\
& g_{k, i}(z \mid x)=N\left(z ; H_{k, i} x, R_{k, i}\right), \quad i=1, \cdots, 4
\end{aligned}
$$

where $N(\cdot ; m, P)$ denotes a Gaussian density with mean $m$ and covariance $P$. $F$ is the state transition matrix, $Q$ is the process noise covariance, $H_{k, i}$ is the observation matrix, and $R_{k, i}$ is the observation noise covariance. The survival and detection probability are assumed as a constant, i.e., $p_{S, k}(x)=p_{S, k}, p_{D, k}(x)=p_{D, k}$. To facilitate the derivation of the closed-form solution, we define an intermediate operator $G_{k, z}^{i}$ by

$$
\left(G_{k, z_{a}}^{i} \phi\right)(x)=g_{k, i}\left(z_{a} \mid x\right) \phi(x)
$$

If $g_{k, i}\left(z_{a} \mid x\right)=N\left(z_{a} ; H_{k, i} x, R_{k, i}\right)$ and $\phi(x)=N\left(z ; m_{\phi}, P_{\phi}\right)$, then $\left(G_{k, z_{a}}^{i} \phi\right)(x)$ is a Gaussian density 


$$
\left(G_{k, z_{a}}^{i} \phi\right)(x)=\rho_{k, i}\left(z_{a}\right) N\left(x ; m_{k, i}, P_{k, i}\right)
$$

where

$$
\begin{aligned}
& \xi_{k \mid k-1, i}=H_{k, i} m_{\phi} \\
& S_{k \mid k-1, i}=R_{k, i}+H_{k, i} P_{\phi} H_{k, i}^{T} \\
& \rho_{k, i}\left(z_{a}\right)=N\left(z_{a} ; \xi_{k \mid k-1, i} S_{k \mid k-1, i}\right) \\
& m_{k, i}=m_{\phi}+K_{k, i}\left(z_{a}-\xi_{k \mid k-1, i}\right) \\
& P_{k, i}=\left(I-K_{k, i} H_{k, i}\right) P_{\phi} \\
& K_{k, i}=P_{\phi} H_{k, i}^{T}\left(S_{k \mid k-1, i}\right)^{-1}
\end{aligned}
$$

We suppose that the predicted PHD filter at time $k-1$ has the following GM representation:

$$
\begin{aligned}
D_{k \mid k-1}(x) & =\sum_{l=1}^{J_{k \mid k-1}} \omega_{k \mid k-1}^{(l)} \cdot N\left(x ; m_{k \mid k-1}^{(l)}, P_{k \mid k-1}^{(l)}\right) \\
& =\sum_{l=1}^{J_{k \mid k-1}} \omega_{k \mid k-1}^{(l)} \cdot \phi_{k \mid k-1}^{(l)}(x)
\end{aligned}
$$

where $\omega_{k \mid k-1}^{(l)}$ is the weight of the $l$ th component and $J_{k \mid k-1}$ is the predicted number of the components. Then, the update equation at time $k$ can be expressed as a GM of

$$
D_{k \mid k}(x)=D_{k \mid k}^{N D}(x)+\sum_{\wp<Z_{k}} \sum_{W \in\{\wp} D_{k \mid k}^{D}(x, W)
$$

The GM components $D_{k \mid k}^{N D}(x)$, handling the no detections cases, are given by

$$
\begin{aligned}
& D_{k \mid k}^{N D}(x)=\sum_{l=1}^{J_{k \mid k-1}} \omega_{k \mid k}^{(l)} \cdot N\left(x ; m_{k \mid k}^{(l)}, P_{k \mid k}^{(l)}\right) \\
& \omega_{k \mid k}^{(l)}=q_{D, k}^{4} \omega_{k \mid k-1}^{(l)} \\
& m_{k \mid k}^{(l)}=m_{k \mid k-1}^{(l)} \\
& P_{k \mid k}^{(l)}=P_{k \mid k-1}^{(l)}
\end{aligned}
$$

The GM components $D_{k \mid k}^{D}(x, W)$, handling detected target cases, are given by

$$
D_{k \mid k}^{D}(x)=\sum_{l=1}^{J_{k \mid k-1}} \omega_{k \mid k}^{(l)} \cdot N\left(x ; m_{k \mid k}^{(l)}, P_{k \mid k}^{(l)}\right)
$$

$$
\begin{aligned}
& \omega_{k \mid k}^{(l)}=\omega_{\wp} \cdot\left(\frac{b_{W}}{d_{W}}\right)^{(l)} \cdot \omega_{k \mid k-1}^{(l)} \\
& \omega_{\wp}=\frac{\prod_{W \in \wp^{\prime}} d_{W}}{\sum_{\wp^{\prime} \angle Z_{k}} \prod_{W \in \wp^{\prime}} d_{W}}
\end{aligned}
$$

when $W=\left\{z_{1}\right\}$,

$$
\begin{gathered}
d_{W}=1+\sum_{l=1}^{J_{k \mid k-1}} \frac{q_{D, k}^{3} p_{D, k}}{\lambda c\left(z_{1}\right)} \omega_{k \mid k-1}^{(l)} \sum_{i=1}^{4}\left(G_{k, z_{1}}^{i} \phi_{k \mid k-1}^{(l)}\right)(x) \\
\left(\frac{b_{W}}{d_{W}}\right)^{(l)}=\frac{q_{D, k}{ }^{3} p_{D, k} \sum_{i=1}^{4}\left(G_{k, z_{1}}^{i} \phi_{k \mid k-1}^{(l)}\right)(x)}{\lambda c\left(z_{1}\right)+q_{D, k}{ }^{3} p_{D, k} \sum_{r=1}^{J_{k \mid k-1}} \omega_{k \mid k-1}^{(r)} \sum_{i=1}^{4}\left(G_{k, z_{1}}^{i} \phi_{k \mid k-1}^{(r)}\right)(x)}
\end{gathered}
$$

when $W=\left\{z_{1}, z_{2}\right\}$

$$
\begin{gathered}
d_{W}=\frac{q_{D, k}{ }^{2} p_{D, k}{ }^{2}}{\lambda c\left(z_{1}\right) \lambda c\left(z_{2}\right)} \sum_{l=1}^{J_{k \mid k-1}} \omega_{k \mid k-1}^{(l)} \sum_{i=1}^{3} \sum_{j=i+1}^{4} \sum_{a=1}^{2} \sum_{\substack{b=1 \\
a \neq b}}^{2}\left(G_{k, z_{a}}^{i}{ }^{\circ} G_{k, z_{b}}^{j} \phi_{k \mid k-1}^{(l)}\right)(x) \\
\left(\frac{b_{W}}{d_{W}}\right)^{(l)}=\frac{\sum_{i=1}^{3} \sum_{j=i+1}^{4} \sum_{a=1}^{2} \sum_{\substack{b=1 \\
a \neq b}}^{2}\left(G_{k, z_{a}}^{i}{ }^{\circ} G_{k, z_{b}}^{j} \phi_{k \mid k-1}^{(l)}\right)(x)}{\sum_{r=1}^{J_{k \mid k-1}} \omega_{k \mid k-1}^{(r)} \sum_{i=1}^{3} \sum_{j=i+1}^{4} \sum_{a=1}^{2} \sum_{\substack{b=1 \\
a \neq b}}^{2}\left(G_{k, z_{a}}^{i}{ }^{\circ} G_{k, z_{b}}^{j} \phi_{k \mid k-1}^{(r)}\right)(x)}
\end{gathered}
$$

when $W=\left\{z_{1}, z_{2}, z_{3}\right\}$

$$
\begin{aligned}
& d_{W}=\frac{q_{D, k} p_{D, k}{ }^{3}}{\lambda c\left(z_{1}\right) \lambda c\left(z_{2}\right) \lambda c\left(z_{3}\right)} \sum_{l=1}^{J_{k \mid k-1}} \omega_{k \mid k-1}^{(l)} \sum_{i=1}^{2} \sum_{j=i+1}^{3} \sum_{m=j+1}^{4} \sum_{a=1}^{3} \sum_{b=1}^{3} \sum_{c=1}^{3} \\
& \begin{array}{rl}
a \neq b & a \neq c \\
& b \neq c
\end{array} \\
& \times\left(G_{k, z_{a}}^{i}{ }^{\circ} G_{k, z_{b}}^{j}{ }^{\circ} G_{k, z_{c}}^{m} \phi_{k \mid k-1}^{(l)}\right)(x) \\
& \sum_{i=1}^{2} \sum_{j=i+1}^{3} \sum_{m=j+1}^{4} \sum_{a=1}^{3} \sum_{b=1}^{3} \sum_{c=1}^{3}\left(G_{k, z_{a}}^{i} \cdot G_{k, z_{b}}^{j} \cdot G_{k, z_{c}}^{m} \phi_{k \mid k-1}^{(l)}\right)(x)
\end{aligned}
$$

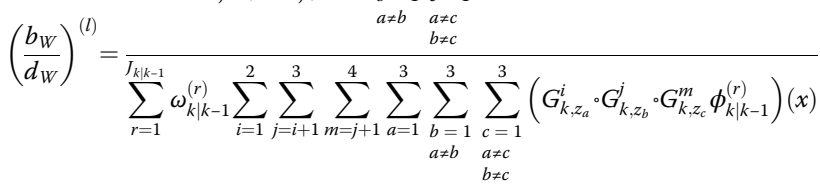

when $W=\left\{z_{1}, z_{2}, z_{3}, z_{4}\right\}$ 


$$
d_{W}=\frac{p_{D, k}{ }^{4}}{\lambda c\left(z_{1}\right) \lambda c\left(z_{2}\right) \lambda c\left(z_{3}\right) \lambda c\left(z_{4}\right)} \sum_{l=1}^{J_{k \mid k-1}} \omega_{k \mid k-1}^{(l)} \sum_{i=1} \sum_{j=2} \sum_{m=3} \sum_{n=4} \sum_{a=1}^{4} \sum_{\substack{b=1 \\ a \neq b}}^{4} \sum_{\substack{c=1 \\ a \neq c \\ b \neq c}}^{4} \sum_{\substack{d=1 \\ a \neq d \\ b \neq d \\ c \neq d}}^{4}\left(G_{k, z_{a}}^{i} \cdot G_{k, z_{b}}^{j} \cdot G_{k, z_{c}}^{m} \cdot G_{k, z_{d}}^{n} \phi_{k \mid k-1}^{(l)}\right)(x)
$$

$$
\begin{aligned}
& \sum_{i=1} \sum_{j=2} \sum_{m=3} \sum_{n=4} \sum_{a=1}^{4} \sum_{\substack{b=1 \\
a \neq b}}^{4} \sum_{\substack{c=1 \\
a \neq c}}^{4} \sum_{\substack{d=1 \\
a \neq d}}^{4}\left(G_{k, z_{a}}^{i}{ }^{\circ} G_{k, z_{b}}^{j}{ }^{\circ} G_{k, z_{c}}^{m}{ }^{\circ} G_{k, z_{d}}^{n} \phi_{k \mid k-1}^{(l)}\right)(x)
\end{aligned}
$$

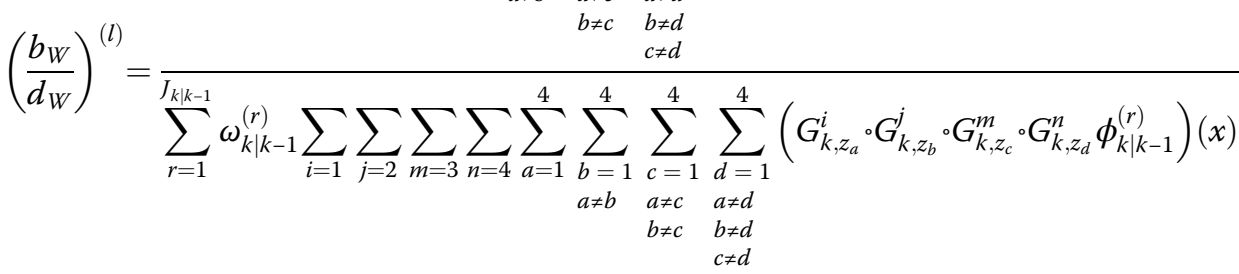

where • denotes a composition.

\subsection{Implementation issues}

1) Nonlinear measurement model: The above Gaussian mixture MP-PHD filter can be extended to a nonlinear measurement model using the EKF. Due to that the dynamic model in the OTHR is linear, we only apply EKF to deal with the nonlinear measurement model in the update step of MP-PHD filter. Use the approximations (55) and (56) in place of the originals (32) and (33), and use the linearizations in (57) to calculate (36) and (37)

$$
\begin{aligned}
& \xi_{k \mid k-1, i}=h_{k, i}\left(m_{\phi}, 0\right) \\
& S_{k \mid k-1, i}=U_{k, i} R_{k, i} U_{k, i}^{T}+H_{k, i} P_{\phi} H_{k, i}^{T}
\end{aligned}
$$

where

$$
H_{k, i}=\left.\frac{\partial h_{k, i}(x, 0)}{\partial x}\right|_{x=m_{\phi}}
$$

2) Managing mixture components: Similar to the standard GM-PHD filter, the techniques of merging and pruning must be used to reduce the exponential growth of the number of Gaussian components, which the merging and pruning methods specific to Gaussian components can be found in [25].

3) Computational complexity: As is shown in Eq. (24), the MP-PHD filter requires all partitions of the current measurements for updating, \& $\angle Z_{k}$ denotes that $\wp$ is one partition of the measurement set $Z_{k}$ and $W \in \wp$ denotes that $W$ is one cell of $\wp$. Note that $W$ does not include null set. For instance, $Z_{k}=\left\{z_{1}, z_{2}, z_{3}\right\}$ can be partitioned as follows [19-21]

$$
\begin{aligned}
& \wp_{1}=\left\{\left\{z_{1}\right\},\left\{z_{2}\right\},\left\{z_{3}\right\}\right\}, \wp_{2}=\left\{\left\{z_{1}, z_{2}\right\},\left\{z_{3}\right\}\right\}, \\
& \wp_{3}=\left\{\left\{z_{1}\right\},\left\{z_{2}, z_{3}\right\}\right\}, \\
& \wp_{4}=\left\{\left\{z_{1}, z_{3}\right\},\left\{z_{2}\right\}\right\}, \wp_{5}=\left\{\left\{z_{1}, z_{2}, z_{3}\right\}\right\},
\end{aligned}
$$

whereas with the number of measurements growing, the number of partitions grows very large, which is computationally infeasible.

From the above analysis, we can see that the computational load required by the proposed MP-PHD filter is similar to that of the extended targets PHD filter [18-22]. Note that some methods, such as K-means++ method [19] and spectral clustering [20], have been suggested for the implementation of the extended targets PHD filter to reduce the number of partitions. However, these methods that applied in the extended targets are measurement dependent. Since the measurement function of each measurement mode can be very different in OTHR. These methods may fail with the multipath tracking problem in OTHR. To limit the number of partitions in the MP-PHD filter, some approximations are necessary in this paper. First, we can use the gating technology with multiple validation gates to reduce the number of measurements for each propagation model before the update step at each time [11], and then we can use the similar method of "effective binary partition" that was implemented in [26] to reduce the number of partitions. For space considerations 
which are not the focus of this paper, we omit the details. Note that we used only a simple partition technique in our implementation, and the development of more efficient implementations will be a future task.

\section{Numerical simulations}

The performance of the proposed MP-PHD filter will be evaluated through two numerical examples, including the single target and multitarget tracking in this section. And we use the optimal subpattern assignment (OSPA) metric to evaluate the performance of the proposed algorithm [27].

\subsection{Experiment 1}

In this subsection, we have designed an experiment to compare the MP-PHD filter with the standard single-path PHD filter in a single target tracking scenario. Note here that the standard single-path PHD filter uses one specific mode (mode EE used in this experiment) to track the targets, and we use the gating technology to reduce the number of measurements for propagation model EE before the update step at each time. The two filters are simulated with the same environment in OTHR. It is assumed that a single nonmaneuvering target is in the presence of clutter. Clutter is generally modeled as a Poisson RFS with the intensity function $\kappa_{k}(y)=\lambda V u(y)$, where $u(\cdot)$ indicates the uniform density over the region $[1000,1400] \mathrm{km} \times[0.069813,0.17453] \mathrm{rad}$, $V=41.8868 \mathrm{~km} \cdot \mathrm{rad}$ is the "volume" of this surveillance region, and $\lambda=4.7743(\mathrm{~km} \cdot \mathrm{rad})^{-1}$ is the average number of the clutter returns per unit volume. This translates to 200 clutter measurements per scan. And each propagation model has the same detection probability and target survive probability that are set to $p_{D, k}=0.6$ and $p_{S, k}=0.95$. To keep computations tractable, the Gaussian component pruning is applied in this numerical example with a pruning threshold $\tau=10^{-5}$, a merging threshold $U=20$, and a maximum number of Gaussian components $J_{\max }=100$. It is assumed that a single target moving in the surveillance region with the initial target state $x_{0}=(1100 \mathrm{~km}, 0.15 \mathrm{~km} / \mathrm{s}$, $0.10472 \mathrm{rad}, 8.72665 \mathrm{e}-05 \mathrm{rad} / \mathrm{s})$. Other simulation parameters are defined as the same as [11]. The true target trajectory and OTHR multipath detections without clutter under $p_{D, k}=0.6$ are shown in Fig. 2. It is shown that the receiver

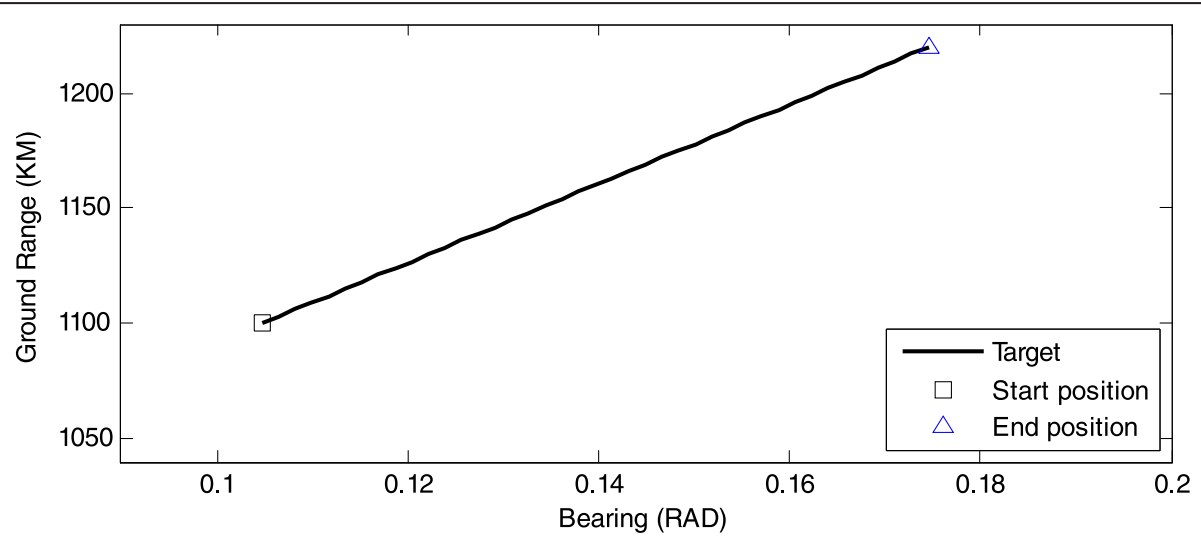

(a) True target trajectory in ground coordinates

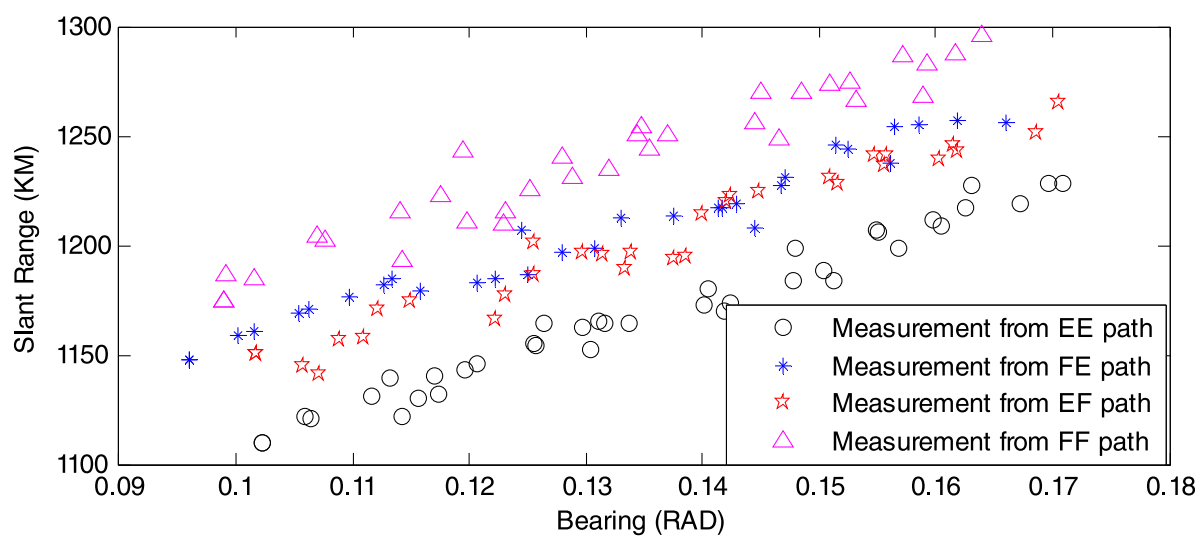

(b) OTHR multipath detections without clutter in slant coordinates

Fig. 2 The scenario of true target trajectory and OTHR multipath detections. a True target trajectory in ground coordinates. b OTHR multipath detections without clutter in slant coordinates 


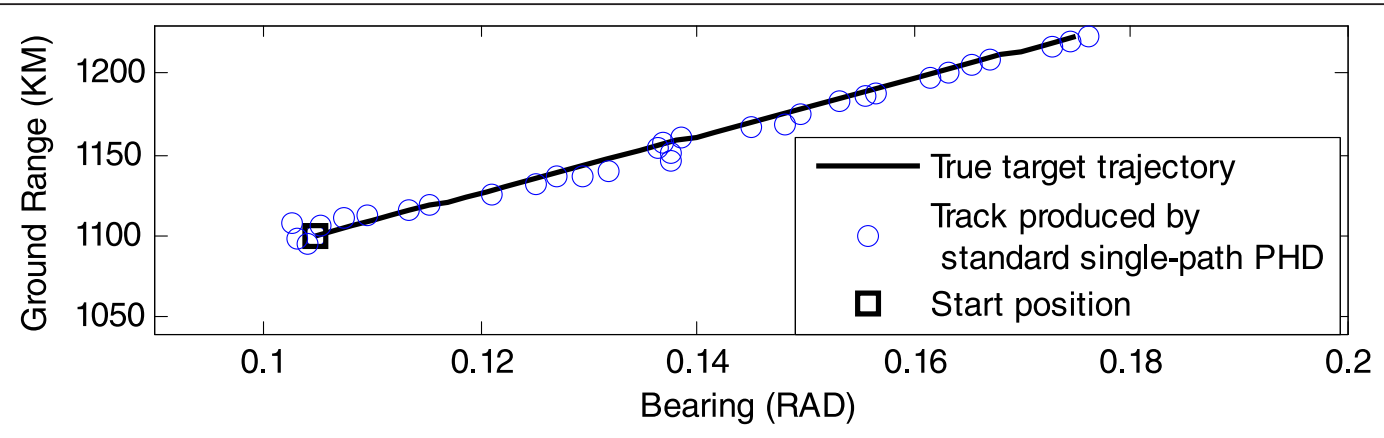

(a) Standard single-path PHD filter

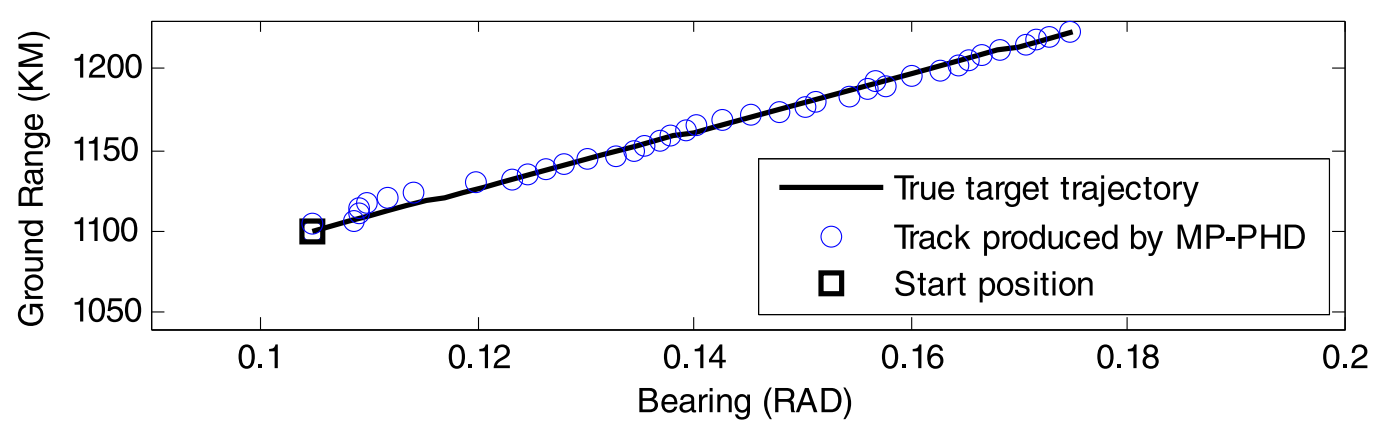

(b) MP-PHD filter

True vs Estimated

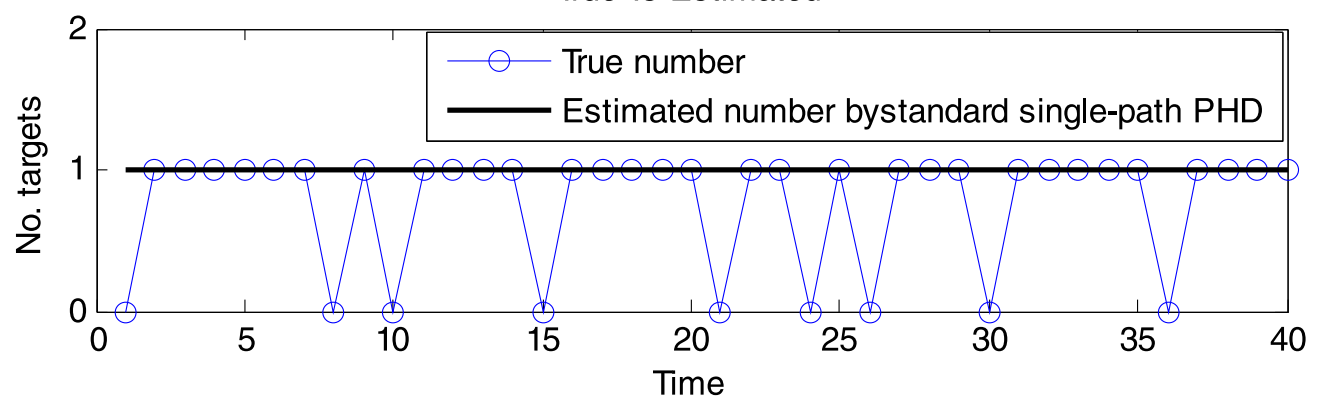

(c) Standard single-path PHD filter

True vs Estimated

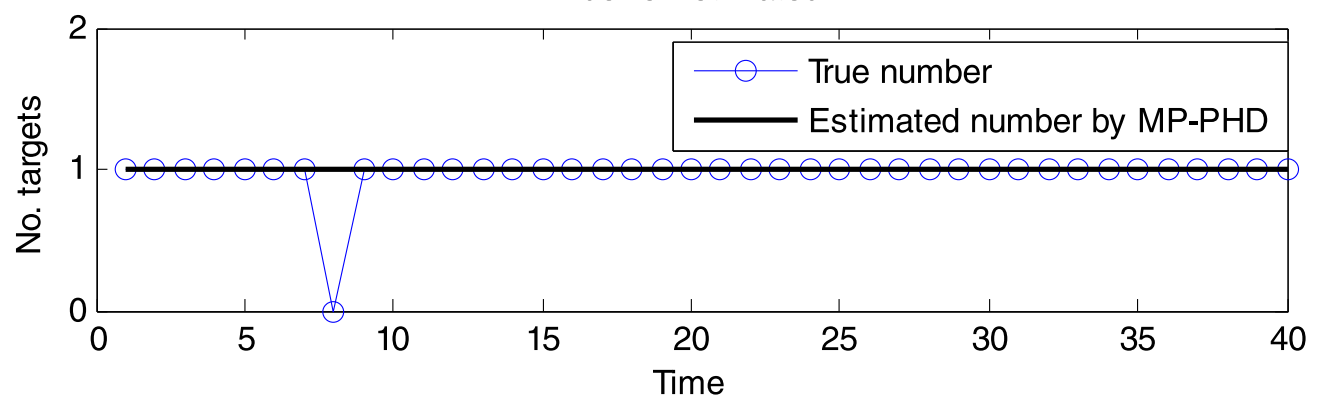

(d) MP-PHD filter

Fig. 3 Target tracking produced by a MP-PHD filter and a single-path PHD filter. a Standard single-path PHD filter. b MP-PHD filter. c Standard single-path PHD filter. d MP-PHD filter 
can obtain more than one measurement from the same target at one scan.

The target tracking results of the MP-PHD filter and the standard single-path PHD filter for one simulation are shown in Fig. 3. As the position estimates are shown in (a) and (b), we can see that the MP-PHD filter provides more accurate position estimates for almost all the time. Moreover, for the simulation results, shown in (c) and (d), using the MP-PHD, there is only one false estimated number at time 8 .

\subsection{Experiment 2}

In this scenario, we assumed that the number of targets is constant. The performance of the GM-MP-PHD filter is compared with the MD-JPDA filter. Two filters are simulated with the same environment in OTHR tracking system, and we also use the gating technology with multiple validation gates to reduce the number of measurements in the MD-JPDA filter. The experiment goes on for $600 \mathrm{~s}$ (sampling period $T=20 \mathrm{~s}$ ), and we assumed that two targets appear with initial state target $1 x_{1}=$ $(1130 \mathrm{~km}, 0.12 \mathrm{~km} / \mathrm{s}, 0.10472 \mathrm{rad}, 8.72665 \mathrm{e}-05 \mathrm{rad} / \mathrm{s})$ and target $2 x_{2}=(1125 \mathrm{~km},-0.1 \mathrm{~km} / \mathrm{s}, 0.11472 \mathrm{rad}$, $7.92665 \mathrm{e}-05 \mathrm{rad} / \mathrm{s})$, respectively. And each propagation model has the same target survive probability and detection probability that are set to $p_{S, k}=0.95$ and $p_{D, k}=0.6$. Other parameters are adopted as the same as the experiment 1 .

The position root mean squared error (RMSE) of the two filters are shown in Fig. 4. It is shown that the proposed MP-PHD filter has a notable performance gain over the MD-JPDA filter. This is due to the fact that the proposed MP-PHD filter encapsulates all the information about the targets by enumerating all of the association between propagation paths and measurements. However, only a subset of the associations (not all the permutations of the propagation paths) is used in the MD-JPDA filter, this is the reason degrades the performance of MD-JPDA filter.

To evaluate the computational time of the proposed algorithm, the averaged time is computed in MATLAB7.1 on an Intel CORE i5 CPU computer with $2 \mathrm{~GB}$ of RAM. The proposed GM-MP-PHD consumed approximately $8.6 \mathrm{~s}$ per $\mathrm{MC}$ run and the MD-JPDA consumed approximately $1.1 \mathrm{~s}$. Although the proposed MP-PHD filter has high computational time, it has better performance than the MD-JPDA filter.

\subsection{Experiment 3}

To validate the tracking performance of MP-PHD filter in multipath multitarget tracking, in this experiment, we have designed a multiple track scenario including three nonmaneuvering targets where the target number is changing in OTHR. The experiment goes on for $800 \mathrm{~s}$ with the sampling period $T=20 \mathrm{~s}$, and it is assumed that target 1 and target 2 appear in the course of the whole experiment with initial state $x_{1}=(1100 \mathrm{~km}, 0.15 \mathrm{~km} / \mathrm{s}, 0.10472 \mathrm{rad}$, $8.72665 \mathrm{e}-05 \mathrm{rad} / \mathrm{s})$ and $x_{2}=(1170 \mathrm{~km},-0.14 \mathrm{~km} / \mathrm{s}$, $0.11472 \mathrm{rad}, 7.72665 \mathrm{e}-05 \mathrm{rad} / \mathrm{s})$, respectively, target 3 appears at $t=180 \mathrm{~s}$ and disappears at $t=480 \mathrm{~s}$ with the initial states $x_{3}=(1170 \mathrm{~km},-0.05 \mathrm{~km} / \mathrm{s}, 0.15701 \mathrm{rad}$, $-8.72665 \mathrm{e}-05 \mathrm{rad} / \mathrm{s})$. In this experiment, there are no spawning targets for simplification. Other simulation conditions are the same as the experiment 1.

1) One simulation: The true target trajectory is shown in Fig. 5, and the position estimates of the MP-PHD filter for one simulation compared with the standard

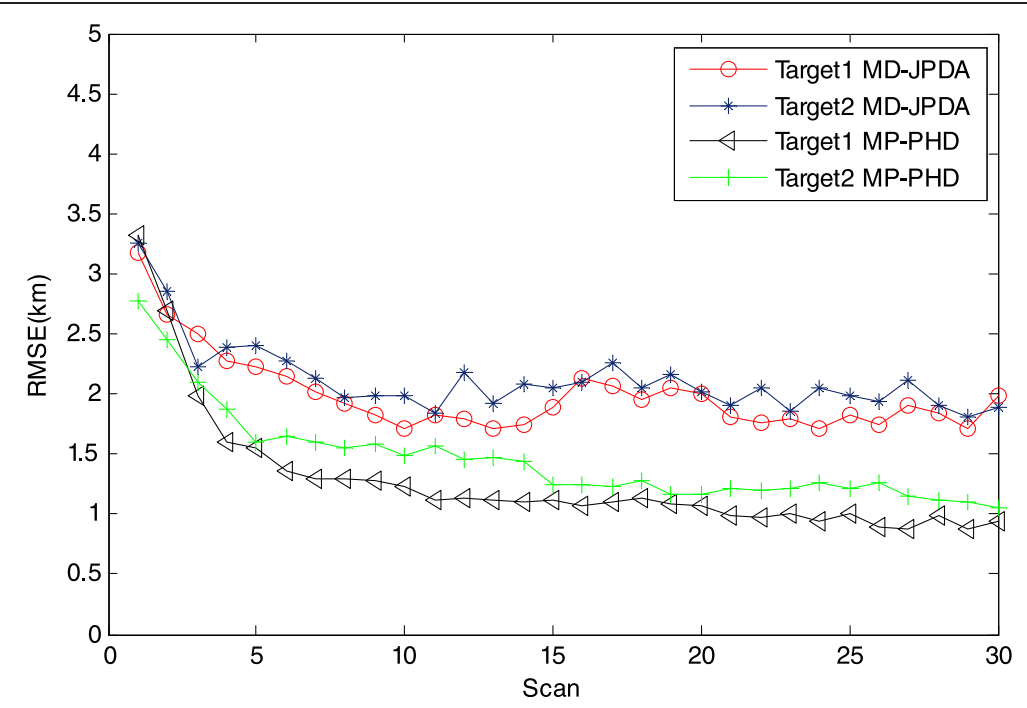

Fig. 4 Position RMSE for a MP-PHD filter and a MD-JPDA filter with OTHR data 


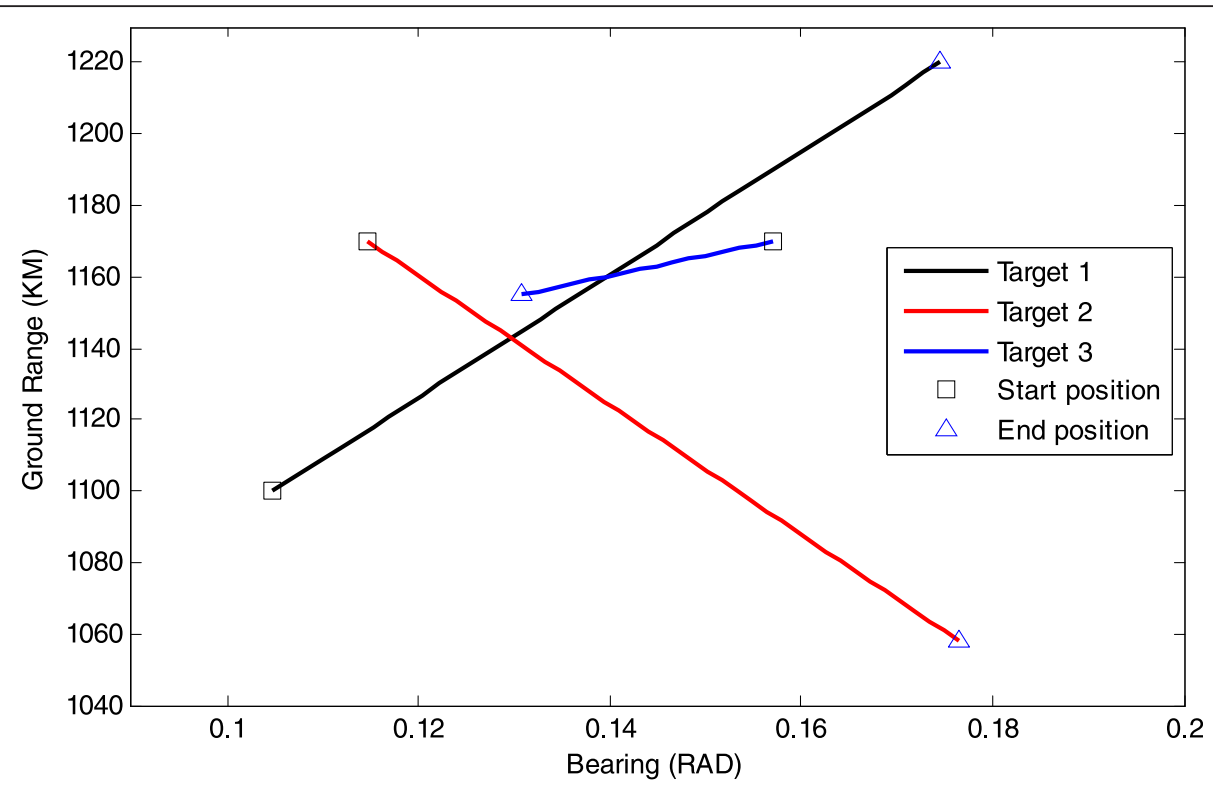

Fig. 5 The true target trajectory

single-path PHD filter are shown in Fig. 6, the estimation of target number is shown in Fig. 7. As shown in Figs. 6 and 7, the MP-PHD filter can accurately estimate the target state and the target number of the multipath multitarget tracking in OTHR. In comparison, the simulation result shows that the MP-PHD filter provides more accurate position estimates for almost all the times.

2) 100 Monte Carlo simulations: We compare the MPPHD filter with the standard single-path PHD filter over 100 Monte Carlo simulations with different detection probability $p_{D, k}=0.6$ and $p_{D, k}=0.99$, respectively. We use the OSPA metric to evaluate the performance, the parameters of OSPA distance are set to the cutoff parameter $c=15 \mathrm{~km}$ and the order parameter $p=2$ (see [27] for more details).

Figure 8 shows the average of OSPA distance comparison between the MP-PHD filter and the standard single-path PHD filter, and the average of estimated

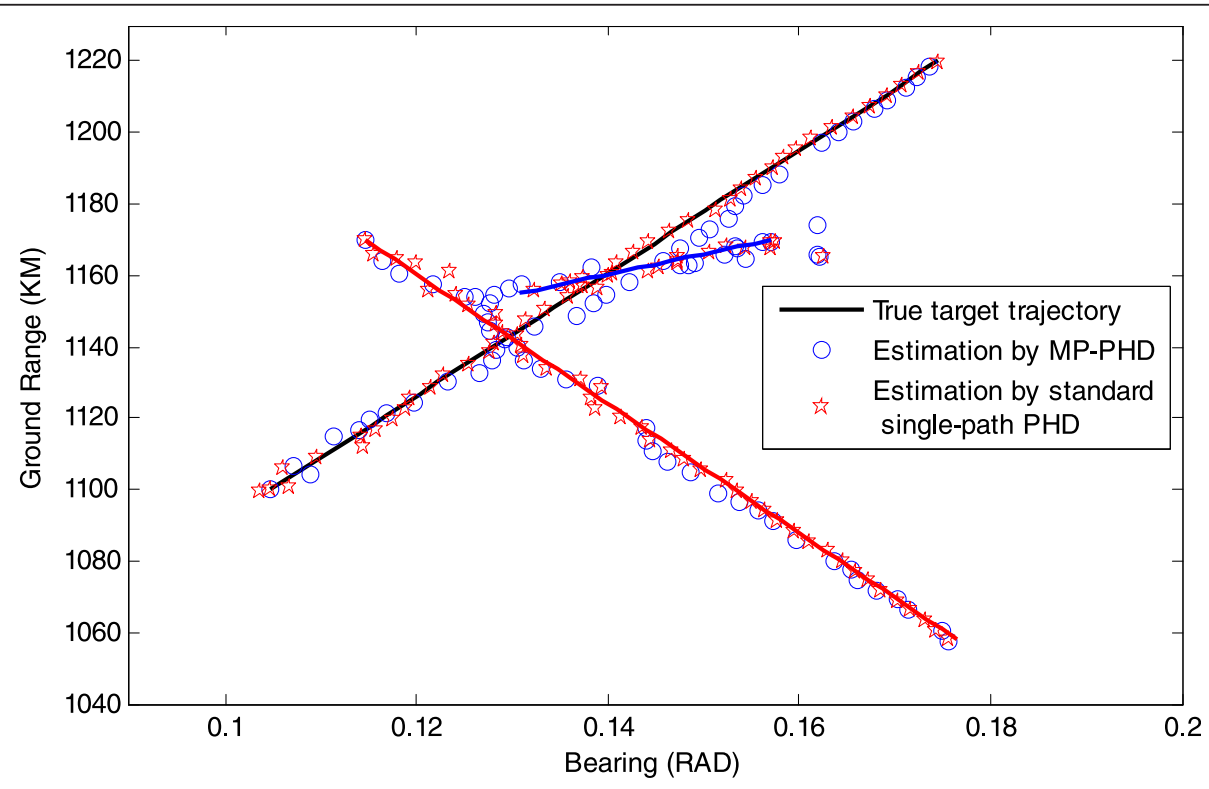

Fig. 6 The true and estimation target trajectory $\left(p_{D, k}=0.6\right)$ 


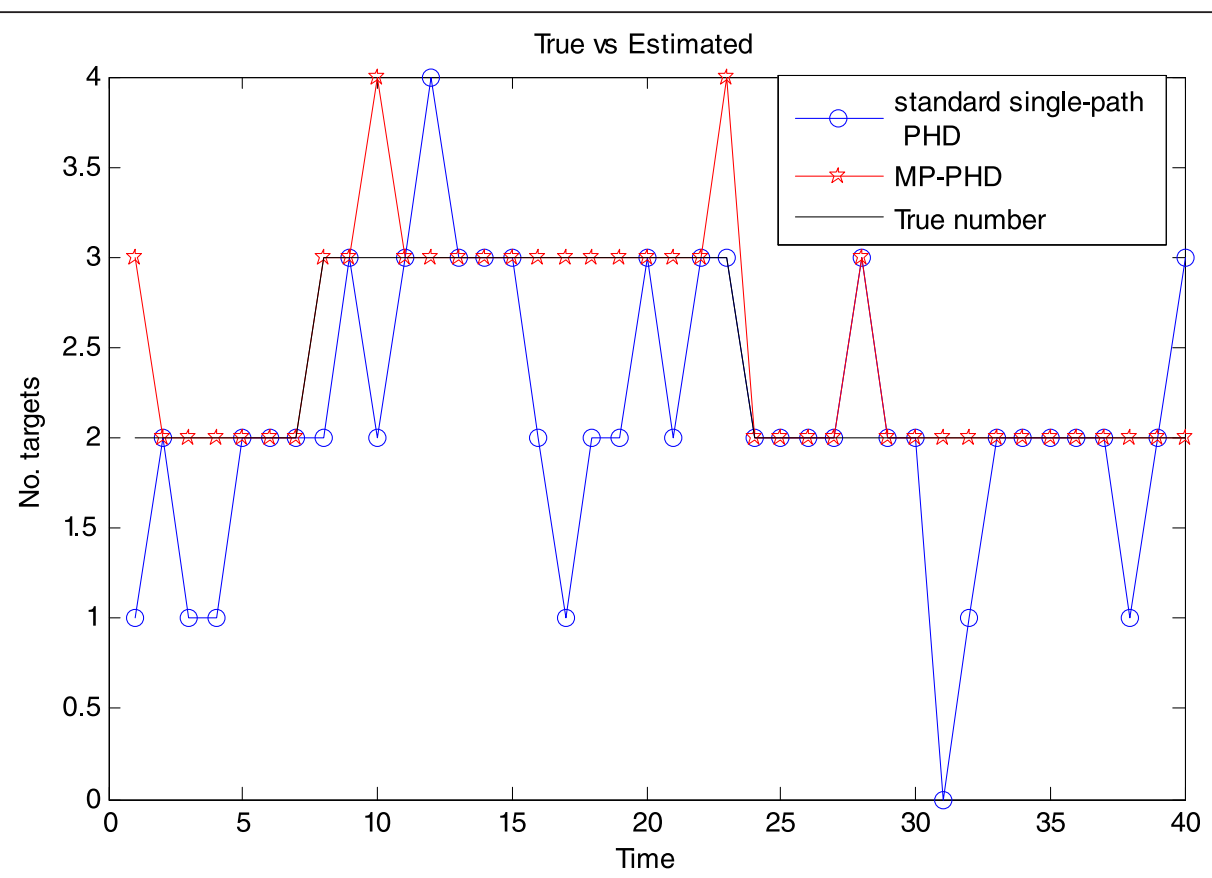

Fig. 7 The true and estimated target number $\left(p_{D, k}=0.6\right)$

number is shown in Fig. 9. The simulation results demonstrate that the tracking performance of the MP-PHD filter surpasses the performance of the standard single-path PHD filter. Moreover, it can accurately estimate the target state and the target number with low detection probability under the multitarget tracking circumstance. As shown in Figs. 8 and 9, the standard single-path PHD filter cannot deal with the multitarget tracking problem effectively under low detection probability. This is due to the fact that the MP-PHD filter use four path measurements to track the targets, and then, it can

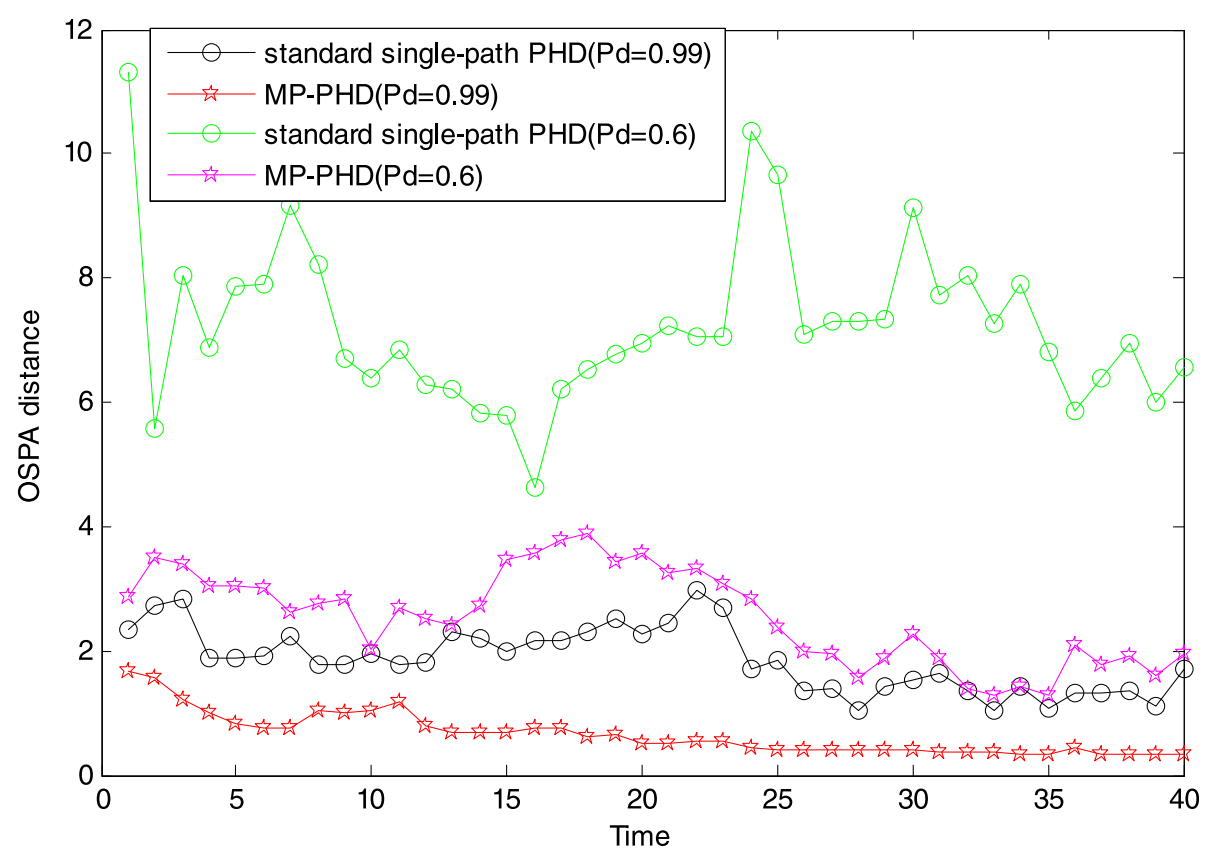

Fig. 8 The average of OSPA distance 


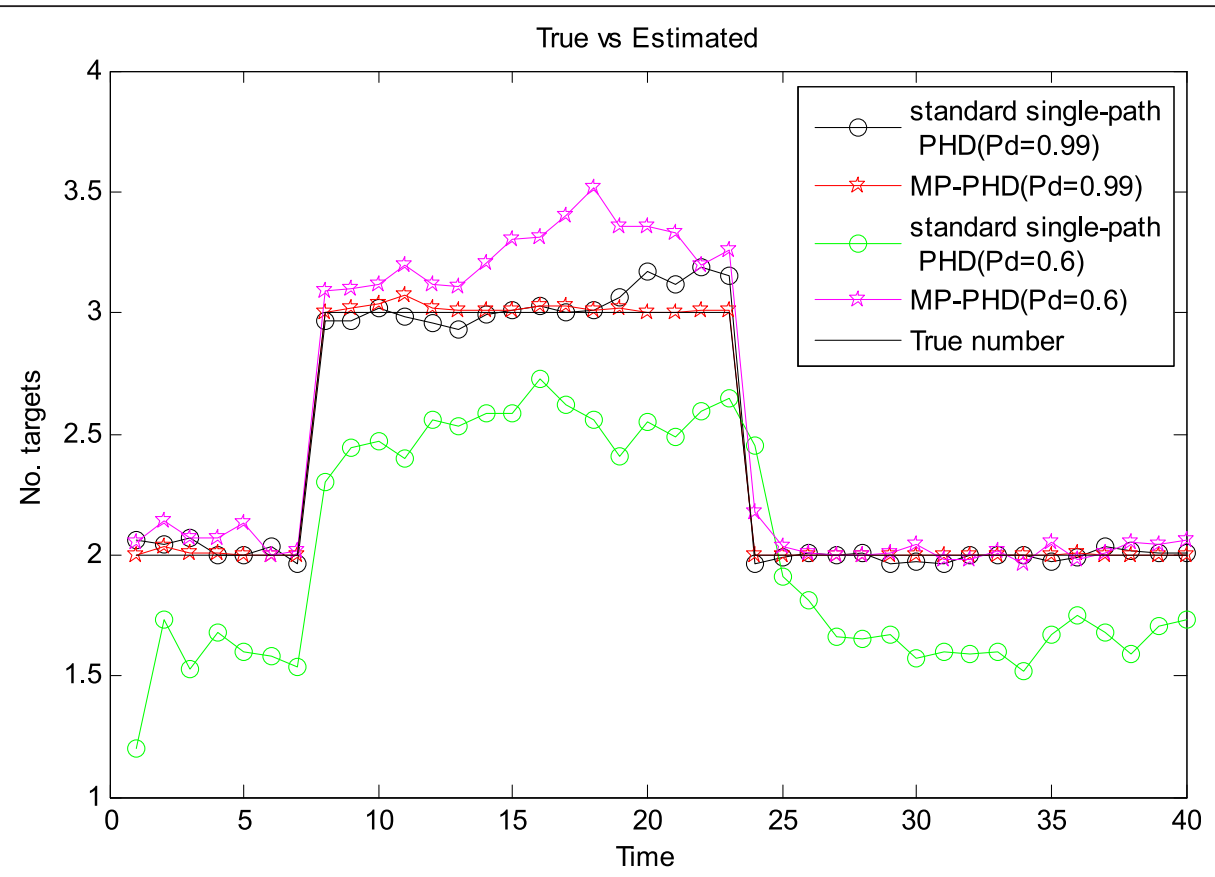

Fig. 9 Average of estimated target number

more effectively use the measurements from the targets under low detection probability.

\section{Conclusions}

In this paper, the MP-PHD filter, which tracks the multipath multitarget in OTHR, has been proposed. The MP-PHD filter is based on the theory of FISST, the FISST is employed to derive the update equation, and then a closed-form solution of MP-PHD filter in the form of GM has been proposed. The EKF is used to deal with the nonlinear problem of the measurement model. Simulation results show that the proposed MP-PHD filter has a notable performance gain over the MD-JPDA filter.

Similar to the standard PHD filter, the MP-PHD filter should be improved for some problems. For example, as the number of targets in the surveillance region is large, the estimated target number is unreliable. To solve this problem, a modified PHD filter name, cardinalized PHD filter, is proposed by Mahlar [28, 29]. Our future work could derive a CPHD filter which can be applied to the multitarget tracking in OTHR.

\section{Appendices}

\subsection{Appendix 1: Proof of Lemma 1}

This lemma is proved by mathematical induction. For the initial induction step, assume $Z=\left\{z_{1}\right\}$. In this case there is only one partition, $\wp=\left\{\left\{z_{1}\right\}\right\}$, and we get

$$
\begin{aligned}
\left.\frac{\delta F}{\delta z_{1}}[g, h]\right|_{g=0}= & F[g, h] \lambda c\left(z_{1}\right)\left(1+\mu s\left[h q_{D, k}^{3} p_{D, k}\left(\sum_{i=1}^{4} \ell_{z_{1}, i}\right)\right]\right. \\
& +\mu s\left[h q_{D, k}^{2} p_{D, k}^{2}\left(\sum_{i=1}^{3} \sum_{j=i+1}^{4}\left(\ell_{z_{1}, i} p_{g, j}+p_{g, i} \ell_{z_{1}, j}\right)\right)\right] \\
& +\mu s\left[h q _ { D , k } p _ { D , k } ^ { 3 } \left(\sum _ { i = 1 } ^ { 2 } \sum _ { j = i + 1 } ^ { 3 } \sum _ { m = j + 1 } ^ { 4 } \left(\ell_{z_{1}, i} p_{g, j} p_{g, m}\right.\right.\right. \\
& \left.\left.\left.\left.+p_{g, i} \ell_{z_{1}, j} p_{g, m}+p_{g, i} p_{g, j} \ell_{z_{1}, m}\right)\right)\right]\right) \\
& +\mu s\left[h p _ { D , k } ^ { 4 } \left(\ell_{z_{1}, 1} p_{g, 2} p_{g, 3} p_{g, 4}+p_{g, 1} \ell_{z_{1}, 2} p_{g, 3} p_{g, 4}\right.\right. \\
& \left.\left.\left.+p_{g, 1} p_{g, 2} \ell_{z_{1}, 3} p_{g, 4}+p_{g, 1} p_{g, 2} p_{g, 3} \ell_{z_{1}, 4}\right)\right]\right)\left.\right|_{g=0} \\
= & F[0, h] \lambda c\left(z_{1}\right)\left(1+\mu s\left[h q_{D, k}^{3} p_{D, k}\left(\sum_{i=1}^{4} \ell_{z_{1}, i}\right)\right]\right) \\
= & F[0, h] \lambda c\left(z_{1}\right) d_{\left\{z_{1}\right\}}[0, h]
\end{aligned}
$$

Now, assume that we have established Eq. (18) for $Z=\left\{z_{1}, \cdots, z_{m}\right\}$ with $|Z|=m>1$. We are to establish Eq. (18) for $Z=\left\{z_{1}, \cdots, z_{m}, z_{m+1}\right\}$ and $|Z|=m+1$. Using the product rule for functional derivatives, we get

$$
\begin{aligned}
& \left.\frac{\delta^{2} F}{\delta Z \delta z_{m+1}}[g, h]\right|_{g=0}=\left.\frac{\delta F}{\delta z_{m+1}}[g, h]\right|_{g=0} \cdot \prod_{Z} \cdot \sum_{\wp \wp Z Z}\left(\prod_{W \in \mathfrak{\wp}} d_{W}[0, h]\right)
\end{aligned}
$$

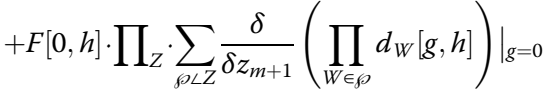

On the one hand, 


$$
\left.\frac{\delta F}{\delta z_{m+1}}[g, h]\right|_{g=0}=F[0, h] \lambda c\left(z_{m+1}\right) d_{\left\{z_{m+1}\right\}}[0, h]
$$

On the other hand,

$\left.\frac{\delta}{\delta z_{m+1}} \prod_{W \in \mathfrak{G}} d_{W}[g, h]\right|_{g=0}=\left.\left(\prod_{W \in \mathfrak{G}} d_{W}[0, h]\right) \cdot \sum_{W \in \mathfrak{S}^{\prime}} \frac{1}{d_{W}[0, h]} \frac{\delta d_{W}}{\delta z_{m+1}}[g, h]\right|_{g=0}$

where

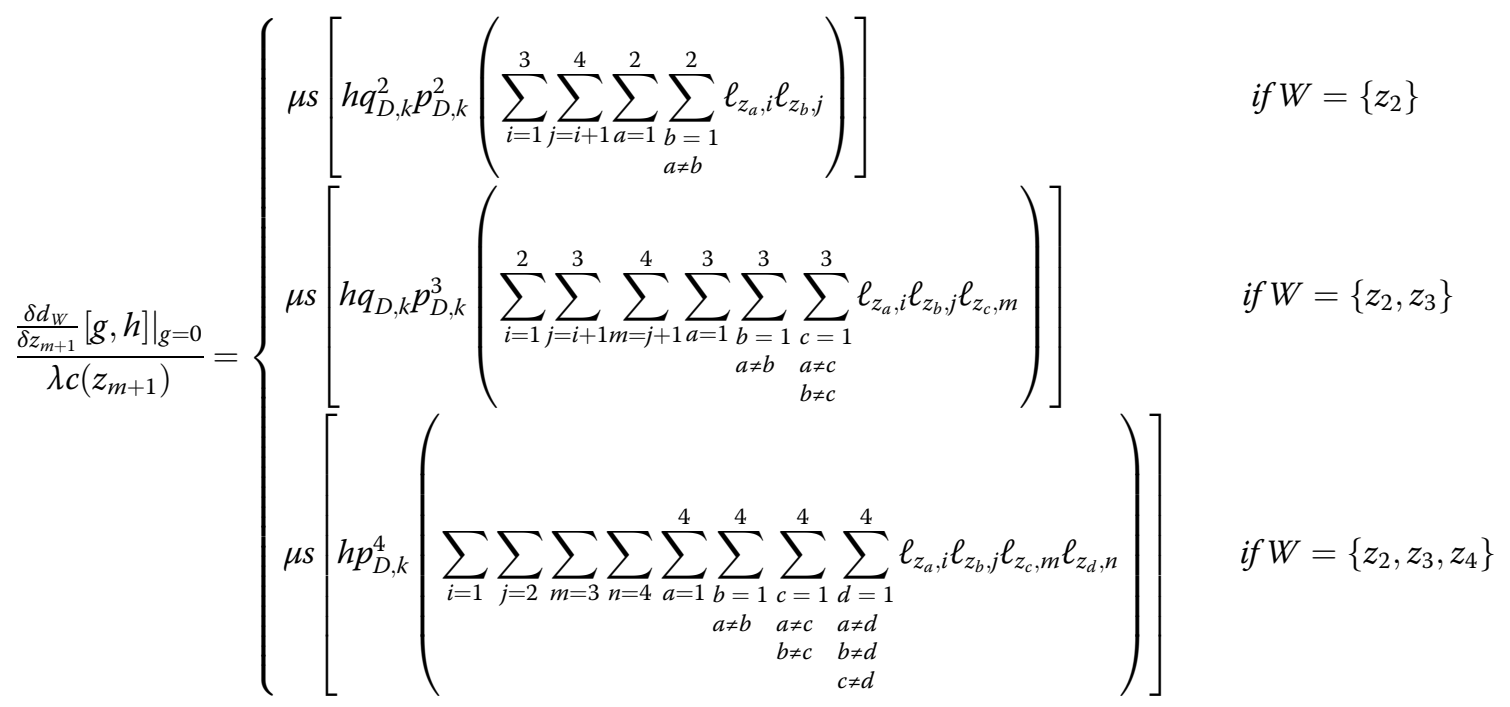

In the above equation, we use $z_{1}$ to replace $z_{m+1}$ for simplicity. And it is easy to find that $\frac{\delta F}{\delta z_{m+1}}[g, h] \times$ $\left.\right|_{g=0}=\lambda c\left(z_{m+1}\right) d_{W \cup\left\{z_{m+1}\right\}}[0, h]$. Putting this all together, Eq. (59) can be written as

$$
\begin{aligned}
& \left.\frac{\delta^{2} F}{\delta Z \delta z_{m+1}}[g, h]\right|_{g=0}=F[0, h] \cdot \prod_{Z \cup\left\{z_{m+1}\right\}} \cdot \sum_{\mathscr{f}<Z}\left(\prod_{W \in\left\{\mathfrak { S } \left\{\left\{\left\{z_{m+1}\right\}\right\}\right.\right.} d_{W}[0, h]\right) \\
& +F[0, h] \cdot \prod_{Z \cup\left\{z_{m+1}\right\}} \cdot \sum_{\mathfrak{\wp}<Z}\left(\prod_{W \in \mathfrak{\wp}} d_{W}[0, h]\right) . \\
& \left(\sum_{W \in\{} \frac{d_{W \cup\left\{z_{m+1}\right\}}[0, h]}{d_{W}[0, h]}\right)
\end{aligned}
$$

Note that all partitions of the $Z \cup\left\{z_{m+1}\right\}$ have the following forms. First, take a partition $\wp$ of the set $Z$ and add the cell $\left\{z_{m+1}\right\}$ to obtain a new partition $\wp_{1}\left(z_{m+1}\right)=\wp \cup\left\{\left\{z_{m+1}\right\}\right\}$ of $Z \cup\left\{z_{m+1}\right\}$. This action is what is denoted mathematically in the first line of Eq. (63). Secondly, remove a cell $W$ from the partition $\wp$ and replace it with $W \cup\left\{z_{m+1}\right\}$ of a new partition $\wp_{2}\left(z_{m+1}, W\right)=\left\{W \cup\left\{z_{m+1}\right\}\right\} \cup \cup \cup_{V \in \wp-\{W\}}\{V\}$ of $Z \cup\left\{z_{m+1}\right\}$. This action is what is denoted by the product

$$
\left(\prod_{W \in\{} d_{W}[0, h]\right) \cdot\left(\sum_{W \in\{} \frac{d_{W \cup\left\{z_{m+1}\right\}}[0, h]}{d_{W}[0, h]}\right)
$$

in the second line of Eq. (63). Consequently, Eq. (63) becomes

$$
\left.\frac{\delta^{2} F}{\delta Z \delta z_{m+1}}[g, h]\right|_{g=0}=F[0, h] \cdot \prod_{Z \cup\left\{z_{m+1}\right\}} \cdot \sum_{\wp\left\llcorner Z \cup\left\{z_{m+1}\right\}\right.}\left(\prod_{W \in \mathfrak{\wp}} d_{W}[0, h]\right)
$$

and this ends the inductive step.

\subsection{Appendix 2: Proof of Lemma 2}

From Eq. (10), we get 


$$
\begin{aligned}
G_{k \mid k}[h] & =\frac{\frac{\delta F}{\delta Z_{k}}[0, h]}{\frac{\delta F}{\delta Z_{k}}[0,1]} \\
& =\frac{F[0, h]}{F[0,1]} \cdot \frac{\sum_{\wp<Z_{k}} \prod_{W \in \wp} d_{W}[0, h]}{\sum_{\wp^{\prime} \angle Z_{k}} \prod_{W \in \wp^{\prime}} d_{W}[0,1]} \\
& =\exp \left(\mu s\left[(h-1) q_{D, k}^{4}\right]\right) \cdot \frac{\sum_{\wp\left\llcorner Z_{k}\right.} \prod_{W \in \wp^{\prime}} d_{W}[0, h]}{\sum_{\wp^{\prime} \angle Z_{k}} \prod_{W \in \wp^{\prime}} d_{W}[0,1]}
\end{aligned}
$$

\subsection{Appendix 3: Proof of proposition 1}

The posterior multipath PHD filter can be calculated as

$$
D_{k \mid k}(x)=\left.\frac{\delta G_{k \mid k}}{\delta x}[h]\right|_{h=1}
$$

Thus, from Eq. (21), we must first determine

$$
\begin{aligned}
\frac{\delta G_{k \mid k}}{\delta x}[h]= & \frac{\delta F_{0}}{\delta x}[h] \cdot \frac{\sum_{\wp\left\llcorner Z_{k}\right.} \prod_{W \in \wp} d_{W}[0, h]}{\sum_{\wp^{\prime}\left\llcorner Z_{k}\right.} \prod_{W \in \wp^{\prime}} d_{W}[0,1]} \\
& +F_{0}[h] \cdot \frac{\sum_{\wp\left\llcorner Z_{k}\right.} \frac{\delta}{\delta x} \prod_{W \in \wp^{\prime}} d_{W}[0, h]}{\sum_{\wp^{\prime} \angle Z_{k}} \prod_{W \in \wp^{\prime}} d_{W}[0,1]}
\end{aligned}
$$

On the one hand, use Eq. (22) and get

$$
\frac{\delta F_{0}}{\delta x}[h]=F_{0}[h] \cdot D_{k \mid k-1}(x) \cdot q_{D, k}^{4}(x)
$$

On the other hand,

$$
\frac{\delta}{\delta x} \prod_{W \in \wp} d_{W}[0, h]=\prod_{W \in \wp} d_{W}[0, h] \sum_{W \in \wp} \frac{\frac{\delta d_{W}}{\delta x}[0, h]}{d_{W}[0, h]}
$$

where from Eq. (19), we have

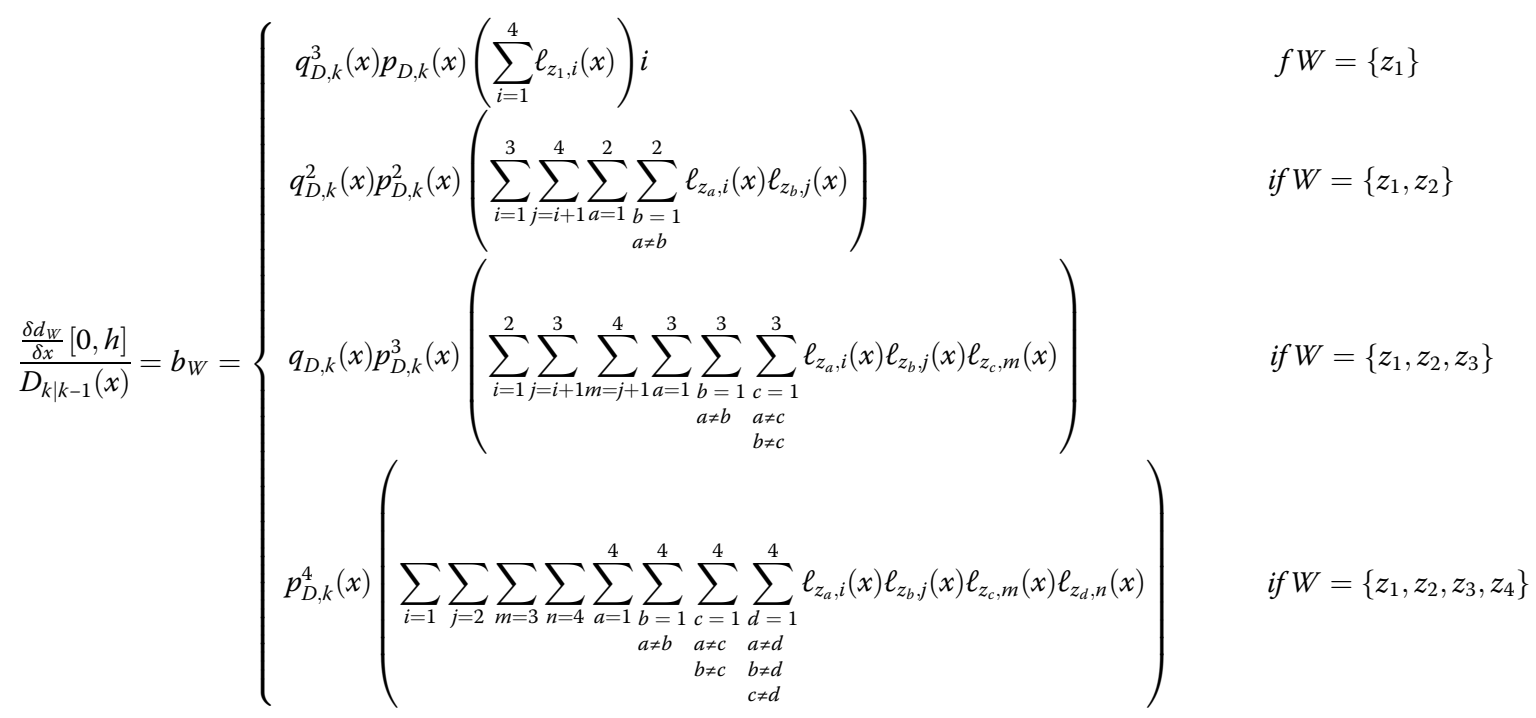




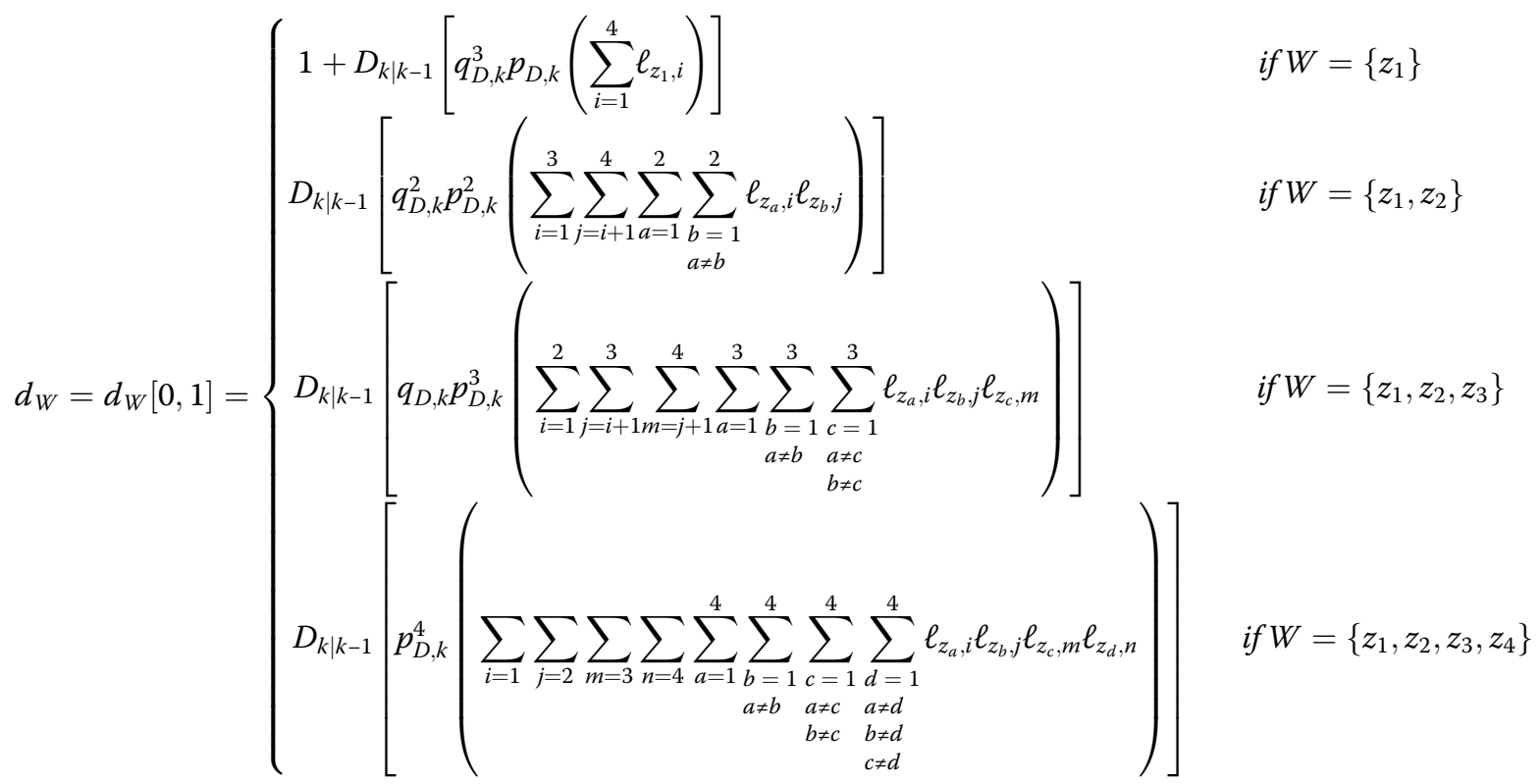

\section{Competing interests}

The authors declare that they have no competing interests.

\section{Author details}

'School of Electronic Information and Communications, Huazhong University of Science and Technology, Wuhan 430074, China. ${ }^{2}$ School of Electrical and Information Engineering, Wuhan Institute of Technology, Wuhan 430073, China.

Received: 10 March 2015 Accepted: 2 December 2015

\section{Published online: 24 December 2015}

\section{References}

1. DJ Percival and KAB White, Multipath track fusion for over-the-horizon radar, in: Proceedings of SPIE Signal Data Process of Small Targets, San Diego, CA, United States, July 27, 1997, pp. 363-374.

2. DJ Percival and KAB White, Multihypothesis fusion of multipath over-thehorizon radar tracks, in: Proceedings of SPIE Signal Data Process of Small Targets, Orlando, FL, April 13, 1998, pp. 440-451.

3. MG Rutten and D J Percival, Joint lonospheric and Target State Estimation for Multipath OTHR Track Fusion, in: Proceedings of SPIE Signal Data Process of Small Targets, vol. 4473, San Diego, CA, USA, July 29, 2001, pp. 118-129.

4. GW Pulford, OTHR multipath tracking with uncertain coordinate registration. IEEE Transactions on Aerospace and Electronic Systems 40(41), 38-56 (2004)

5. H Liu, Q Pan, Y Liang, Y Cheng, M Cheng, Comments on "multipath data association tracker for over-the-horizon radar". IEEE Transactions on Aerospace and Electronic Systems 41(3), 1147-1148 (2005)

6. MG Rutten, S Maskell, M Briers and NJ Gordon, Multipath track association for over-the-horizon radar using Lagrangian relaxation, in: Proceedings of SPIE Signal Data Process of Small Targets, Orlando, FL, April 12, 2004, pp. 452-463.

7. Y Bar-Shalom, E Tse, Tracking in a cluttered environment with probabilistic data association. Automatica 11(5), 451-460 (1975)

8. SB Colegrove, AW Davis, JK Ayliffe, Track initiation and nearest neighbours incorporated into probabilistic data association. Journal Elect. Electronics Eng 6(3), 191-198 (1986)

9. T Kirubarajan, Y Bar-Shalom, Probabilistic data association techniques for target tracking in clutter. Proceedings of the IEEE 92(3), 536-557 (2004)

10. H Liu, Y Liang, Q Pan and Y Cheng, A multipath viterbi data association algorithm for OTHR, in: Proceedings of International Conference on Radar, Shanghai, China, 2006, pp. 1-4
11. GW Pulford, RJ Evans, A multipath data association tracker for over-thehorizon radar. IEEE Transactions on Aerospace and Electronic Systems 34(4), 1165-1183 (1998)

12. SS Blackman, Multiple hypothesis tracking for multiple target tracking. IEEE Aerospace and Electronic Systems Magazine 19(1), 5-18 (2004)

13. HAP Blom, EA Bloem, Interacting multiplemodel joint probabilistic data association, avoiding track coalescence, in Proceedings of the 41th IEEE Conference on Decision Control, vol. 3, 2002, pp. 3408-3415

14. D Musicki, R Evans, Joint integrated probabilistic data association: JPPDA. IEEE Transactions on Aerospace and Electronic Systems 40(3), 1093-1099 (2004)

15. R Mahler, Multi-target Bayes filtering via first-order multi-target moments. IEEE Transactions on Aerospace and Electronic Systems 39(4), 1152-1178 (2003)

16. B Habtemariam, R Tharmarasa, T Thayaparan et al., A multiple-detection joint probabilistic data association filter. IEEE Journal Selected Topics Signal Processing 7(3), 461-471 (2013)

17. R Mahler, "Second-generation" PHD/CPHD filters and multitarget calculus, in: Proceedings of SPIE Signal Data Process of Small Targets, San Diego, CA, August 02, 2009.

18. R Mahler, PHD filters for nonstandard target, l: extended targets, in: Proceedings of the 12th International Conference on Information Fusion, Seattle, WA, July 2009, pp. 915-921

19. K Granstrom, C Lundquist, U Orguner, A Gaussian mixture PHD filter for extended target tracking, in: Proceedings of the 13th International Conference on Information Fusion, Edinburgh, UKL, July 2010, pp. 1-8

20. Yang J, Liu F, Ge H, et al., Multiple extended target tracking algorithm based on GM-PHD filter and spectral clustering, EURASIP Journal on Advances in Signal Processing 2014:117

21. Y Zhang, $\mathrm{H} \mathrm{Ji}$, Gaussian mixture reduction based on fuzzy ART for extended target tracking. Signal Process 97, 232-241 (2014)

22. R Mahler, PHD filters for nonstandard targets, II: unresolved targets, in: Proceedings of the 12th International Conference on Information Fusion, Seattle, WA, USA, July 2009 pp. 922-929

23. R Mahler, A El-Fallah, CPHD and PHD filters for unknown backgrounds, III: tractable multitarget filtering in dynamic clutter, in Proceedings of SPIE, vol. 7698,2010

24. R Mahler, CPHD filters for superpositional sensors, in Proceedings of SPIE, vol. 7445,2009

25. B-N Vo, W-K Ma, The Gaussian mixture probability hypothesis density filter. IEEE Transactions on Signal Processing 54(11), 4091-4104 (2006) 
26. J Xu, F Huang, Z Huang, The multi-sensor PHD filter Analytic implementation via Gaussian mixture and effective binary partition, in: Proceedings of the 16th International Conference on Information Fusion, Istanbul, TUR, July 2013 pp. 945-952

27. D Schuhmacher, B-T Vo, B-N Vo, A consistent metric for performance evaluation of multi-object filters. IEEE Transactions on Signal Processing 56(8), 3447-3457 (2008)

28. B-T Vo, B-N Vo, A Cantoni, Analytic implementations of the cardinalized probability hypothesis density filter. IEEE Transactions on Signal Processing 55(7), 3553-3567 (2007)

29. R Mahler, PHD filters of higher order in target number. IEEE Transactions on Aerospace and Electronic Systems 43(4), 1523-1543 (2007)

\section{Submit your manuscript to a SpringerOpen ${ }^{\circ}$ journal and benefit from:}

- Convenient online submission

- Rigorous peer review

- Immediate publication on acceptance

- Open access: articles freely available online

- High visibility within the field

- Retaining the copyright to your article

Submit your next manuscript at $\boldsymbol{s p r i n g e r o p e n . c o m ~}$ 